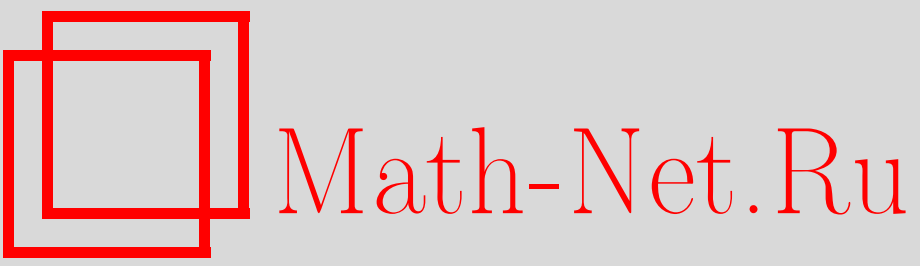

М. А. Скворцова, Оценки решений в модели взаимодействия популяций с несколькими запаздываниями, Итоги науки и техн. Сер. Соврем. мат. и ее прил. Темат. обз., 2020, том 188, 84-105

DOI: https://doi.org/10.36535/0233-6723-2020-188-84-105

Использование Общероссийского математического портала Math-Net.Ru подразумевает, что вы прочитали и согласны с пользовательским соглашением

http: //www.mathnet.ru/rus/agreement

Параметры загрузки:

IP: 54.162 .85 .209

26 апреля 2023 г., 15:50:25 


\title{
ОЦЕНКИ РЕШЕНИЙ \\ В МОДЕЛИ ВЗАИМОДЕЙСТВИЯ ПОПУЛЯЦИЙ \\ С НЕСКОЛЬКИМИ ЗАПАЗДЫВАНИЯМИ
}

\author{
(c) 2020 г. $\quad$ M. А. СКВОРЦОВА
}

\begin{abstract}
АннотАция. Рассматривается система дифференциальных уравнений с несколькими запаздываниями, описывающая взаимодействие $n$ видов микроорганизмов. Получены достаточные условия асимптотической устойчивости нетривиального положения равновесия, соответствующего частичному выживанию популяций. Установлены оценки решений, характеризующие скорость стабилизации на бесконечности, и указаны оценки множества притяжения данного положения равновесия. Результаты получены с использованием модифицированного функционала ЛяпуноваКрасовского.
\end{abstract}

Ключевъе слова: модель взаимодействия популяций, уравнение с запаздывающим аргументом, асимптотическая устойчивость, оценка решения, множество притяжения, модифицированный функционал Ляпунова-Красовского.

\section{ESTIMATES OF SOLUTIONS IN THE MODEL OF INTERACTION OF POPULATIONS WITH SEVERAL DELAYS}

\author{
(c) 2020 M. A. SKVORTSOVA
}

\begin{abstract}
We consider a system of differential equations with several delays, which describes the interaction of $n$ species of microorganisms. We obtain sufficient conditions for the asymptotic stability of a nontrivial equilibrium state corresponding to the partial survival of populations. We establish estimates of solutions that characterize the rate of stabilization at infinity and indicate estimates of the attraction set of a given equilibrium state. The results are obtained by using the modified LyapunovKrasovsky functional.
\end{abstract}

Keywords and phrases: model of interaction of populations, equation with retarded argument, asymptotic stability, estimate of solution, attraction set, modified Lyapunov-Krasovsky functional.

AMS Subject Classification: 34K20, 34K60, 92D25

1. Предварительные сведения. В настоящей работе мы продолжаем исследование системы дифференциальных уравнений с запаздываниями следующего вида:

$$
\left\{\begin{array}{l}
\frac{d}{d t} S(t)=\left(S^{0}-S(t)\right) D-\sum_{i=1}^{n} p_{i}(S(t)) N_{i}(t), \\
\frac{d}{d t} N_{i}(t)=-D_{i} N_{i}(t)+\alpha_{i} p_{i}\left(S\left(t-\tau_{i}\right)\right) N_{i}\left(t-\tau_{i}\right), \quad i=1,2, \ldots, n .
\end{array}\right.
$$

Работа выполнена при поддержке Российского фонда фундаментальных исследований (проекты № 18-31-00408, № 18-29-10086). 
Система описывает взаимодействие $n$ видов микроорганизмов (см. [14]), при этом $N_{i}(t)$ - численность популяции $i$-го вида, $S(t)$ - концентрация питательного вещества. Коэффициенты системы $S^{0}, D, D_{i}, \alpha_{i}$ предполагаются положительными и постоянными, параметры запаздывания $\tau_{i} \geqslant 0$ также предполагаются постоянными. Предполагается, что $p_{i}(S)$ - локально липшицевы, монотонно возрастающие функции и $p_{i}(0)=0$. Более подробное описание модели содержится в [14].

Для системы (1.1) зададим начальные условия:

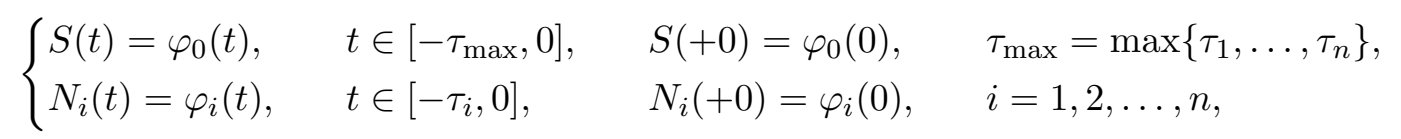

где $\varphi_{0}(t), \varphi_{1}(t), \ldots, \varphi_{n}(t)$ - непрерывные неотрицательные функции. Хорошо известно, что решение начальной задачи (1.1)-(1.2) существует и единственно. Более того, как было отмечено в [14], при неотрицательных начальных функциях решение задачи (1.1)-(1.2) определено на всей правой полуоси $\{t>0\}$, имеет неотрицательные компоненты, при этом каждая компонента решения является ограниченной функцией.

Система (1.1) имеет тривиальное положение равновесия

$$
S(t)=S^{0}, \quad N_{i}(t)=0, \quad i=1,2, \ldots, n .
$$

В [14] было показано, что при выполнении неравенств $\alpha_{i} p_{i}\left(S^{0}\right)<D_{i}, i=1,2, \ldots, n$, все решения уравнения (1.1) с неотрицательными начальными условиями стабилизируются на бесконечности к данному положению равновесия. В [10] были получены оценки решений, характеризующие скорость стабилизации на бесконечности к положению равновесия (1.3). При получении оценок существенно использовались модифицированные функционалы Ляпунова-Красовского (см. [2, $12,13])$; при этом величины, отвечающие за скорость стабилизации, были указаны конструктивно.

Следуя [14], введем следующие обозначения. Поскольку функции $p_{i}(S)$ являются монотонно возрастающими и $p_{i}(0)=0$, то существуют такие $\lambda_{i} \in(0,+\infty]$, что $p_{i}(S)<D_{i} / \alpha_{i}$ при $S<\lambda_{i}$ и $p_{i}(S)>D_{i} / \alpha_{i}$ при $S>\lambda_{i}$. Отсюда, в частности, следует, что если $\lambda_{i}<+\infty$, то $\lambda_{i}$ удовлетворяет условию $p_{i}\left(\lambda_{i}\right)=D_{i} / \alpha_{i}$.

Заметим, что в случае, если существует такое $\lambda_{i_{0}}$, что $\lambda_{i_{0}}<S^{0}$, система (1.1) имеет нетривиальное положение равновесия:

$$
S(t)=\lambda_{i_{0}}<S^{0}, \quad N_{i_{0}}(t)=\alpha_{i_{0}}\left(S^{0}-\lambda_{i_{0}}\right) D / D_{i_{0}}, \quad N_{i}(t)=0, \quad i \neq i_{0} .
$$

Не ограничивая общности, будем считать, что $i_{0}=1$. Следовательно, нетривиальное положение равновесия будет иметь вид

$$
S(t)=\lambda_{1}<S^{0}, \quad N_{1}(t)=\frac{\alpha_{1}}{D_{1}}\left(S^{0}-\lambda_{1}\right) D, \quad N_{i}(t)=0, \quad i=2,3, \ldots, n .
$$

В [14] были получены достаточные условия на параметры системы (1.1), при которых все решения с неотрицательными начальными условиями стабилизируются на бесконечности к данному положению равновесия.

В настоящей работе мы получим достаточные условия асимптотической устойчивости нетривиального положения равновесия (1.4), установим оценки решений, характеризующие скорость стабилизации на бесконечности, и укажем оценки множества притяжения данного положения равновесия. При получении оценок мы будем использовать модифицированные функционалы Ляпунова-Красовского (см. [2]); при этом все величины, входящие в оценки, будут указаны конструктивно.

2. Устойчивость нетривиального положения равновесия. В данном разделе будем предполагать, что выполнено условие $\lambda_{1}<S^{0}$. В этом случае у системы (1.1) существует нетривиальное положение равновесия (1.4). Дополнительно предположим, что функция $p_{1}(S)$ является непрерывно дифференцируемой в некоторой окрестности точки $S=\lambda_{1}$. Ниже мы получим условия асимптотической устойчивости нетривиального положения равновесия. 
Вначале сведем задачу об устойчивости положения равновесия (1.4) системы (1.1) к задаче об устойчивости нулевого решения. Для этого в системе (1.1) сделаем замену переменных

$$
S(t)=\lambda_{1}+\widetilde{S}(t), \quad N_{1}(t)=\frac{\alpha_{1}}{D_{1}}\left(S^{0}-\lambda_{1}\right) D+\widetilde{N}_{1}(t), \quad N_{i}(t)=\widetilde{N}_{i}(t), \quad i=2,3, \ldots, n .
$$

Тогда, учитывая, что $p_{1}\left(\lambda_{1}\right)=D_{1} / \alpha_{1}$, будем иметь

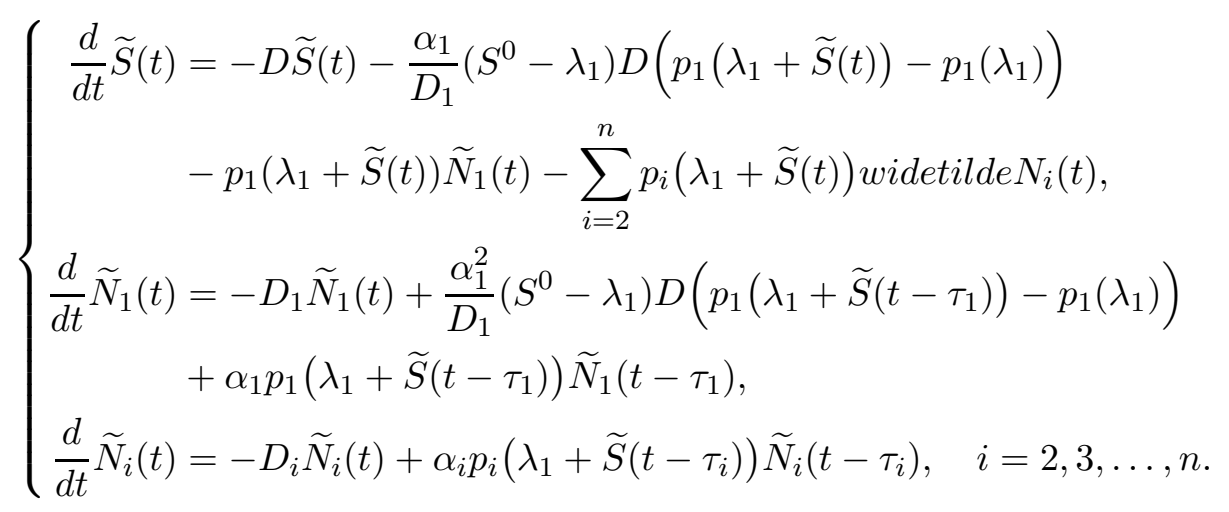

Далее, поскольку функция $p_{1}(S)$ является непрерывно дифференцируемой в некоторой окрестности точки $S=\lambda_{1}$, то систему (2.2) можно переписать в виде

$$
\left\{\begin{aligned}
\frac{d}{d t} \widetilde{S}(t) & =-\left(D+\frac{\alpha_{1}}{D_{1}}\left(S^{0}-\lambda_{1}\right) D p_{1}^{\prime}\left(\lambda_{1}\right)\right) \widetilde{S}(t)-\frac{D_{1}}{\alpha_{1}} \widetilde{N}_{1}(t)-\sum_{i=2}^{n} p_{i}\left(\lambda_{1}\right) \widetilde{N}_{i}(t) \\
& -\frac{\alpha_{1}}{D_{1}}\left(S^{0}-\lambda_{1}\right) D\left(p_{1}\left(\lambda_{1}+\widetilde{S}(t)\right)-p_{1}\left(\lambda_{1}\right)-p_{1}^{\prime}\left(\lambda_{1}\right) \widetilde{S}(t)\right) \\
& -\left(p_{1}\left(\lambda_{1}+\widetilde{S}(t)\right)-p_{1}\left(\lambda_{1}\right)\right) \widetilde{N}_{1}(t)-\sum_{i=2}^{n}\left(p_{i}\left(\lambda_{1}+\widetilde{S}(t)\right)-p_{i}\left(\lambda_{1}\right)\right) \widetilde{N}_{i}(t) \\
\frac{d}{d t} \widetilde{N}_{1}(t) & =-D_{1} \widetilde{N}_{1}(t)+\frac{\alpha_{1}^{2}}{D_{1}}\left(S^{0}-\lambda_{1}\right) D p_{1}^{\prime}\left(\lambda_{1}\right) \widetilde{S}\left(t-\tau_{1}\right)+D_{1} \widetilde{N}_{1}\left(t-\tau_{1}\right) \\
& +\frac{\alpha_{1}^{2}}{D_{1}}\left(S^{0}-\lambda_{1}\right) D\left(p_{1}\left(\lambda_{1}+\widetilde{S}\left(t-\tau_{1}\right)\right)-p_{1}\left(\lambda_{1}\right)-p_{1}^{\prime}\left(\lambda_{1}\right) \widetilde{S}\left(t-\tau_{1}\right)\right) \\
& +\alpha_{1}\left(p_{1}\left(\lambda_{1}+\widetilde{S}\left(t-\tau_{1}\right)\right)-p_{1}\left(\lambda_{1}\right)\right) \widetilde{N}_{1}\left(t-\tau_{1}\right), \\
\frac{d}{d t} \widetilde{N}_{i}(t) & =-D_{i} \widetilde{N}_{i}(t)+\alpha_{i} p_{i}\left(\lambda_{1}\right) \widetilde{N}_{i}\left(t-\tau_{i}\right) \\
& +\alpha_{i}\left(p_{i}\left(\lambda_{1}+\widetilde{S}\left(t-\tau_{i}\right)\right)-p_{i}\left(\lambda_{1}\right)\right) \widetilde{N}_{i}\left(t-\tau_{i}\right), \quad i=2,3, \ldots, n
\end{aligned}\right.
$$

Введем следующие обозначения:

$$
\begin{gathered}
x(t)=\left(\begin{array}{c}
\widetilde{S}(t) \\
\widetilde{N}_{1}(t)
\end{array}\right), \quad y(t)=\left(\begin{array}{c}
\widetilde{S}_{(t)} \\
\widetilde{N}_{1}(t) \\
\widetilde{N}_{2}(t) \\
\vdots \\
\widetilde{N}_{n}(t)
\end{array}\right), \\
A_{1}=\left(\begin{array}{cc}
-\left(D+\gamma_{1}\right) & -D_{1} / \alpha_{1} \\
-D_{1}
\end{array}\right), \quad B_{1}=\left(\begin{array}{cc}
0 & 0 \\
\alpha_{1} \gamma_{1} & D_{1}
\end{array}\right), \quad \gamma_{1}=\frac{\alpha_{1}}{D_{1}}\left(S^{0}-\lambda_{1}\right) D p_{1}^{\prime}\left(\lambda_{1}\right), \\
F_{1}(x(t))=-\left(\begin{array}{c}
f_{a}(x(t))+f_{b}(x(t)) \\
0
\end{array}\right), \\
G_{1}\left(x\left(t-\tau_{1}\right)\right)=\left(\begin{array}{c}
0 \\
\alpha_{1}\left(f_{a}\left(x\left(t-\tau_{1}\right)\right)+f_{b}\left(x\left(t-\tau_{1}\right)\right)\right)
\end{array}\right),
\end{gathered}
$$




$$
\begin{gathered}
f_{a}(x)=\frac{\alpha_{1}}{D_{1}}\left(S^{0}-\lambda_{1}\right) D\left(p_{1}\left(\lambda_{1}+\widetilde{S}\right)-p_{1}\left(\lambda_{1}\right)-p_{1}^{\prime}\left(\lambda_{1}\right) \widetilde{S}\right), \\
f_{b}(x)=\left(p_{1}\left(\lambda_{1}+\widetilde{S}\right)-p_{1}\left(\lambda_{1}\right)\right) \widetilde{N}_{1}, \\
L(y(t))=\left(\begin{array}{c}
l(y(t)) \\
0
\end{array}\right), \quad l(y(t))=-\sum_{i=2}^{n} p_{i}\left(\lambda_{1}\right) \widetilde{N}_{i}(t), \\
U(y(t))=\left(\begin{array}{c}
u(y(t)) \\
0
\end{array}\right), \quad u(y(t))=-\sum_{i=2}^{n}\left(p_{i}\left(\lambda_{1}+\widetilde{S}(t)\right)-p_{i}\left(\lambda_{1}\right)\right) \widetilde{N}_{i}(t) .
\end{gathered}
$$

Тогда система (2.3) запишется в виде

$$
\left\{\begin{aligned}
\frac{d}{d t} x(t) & =A_{1} x(t)+B_{1} x\left(t-\tau_{1}\right)+F_{1}(x(t))+G_{1}\left(x\left(t-\tau_{1}\right)\right)+L(y(t))+U(y(t)), \\
\frac{d}{d t} \widetilde{N}_{i}(t) & =-D_{i} \widetilde{N}_{i}(t)+\alpha_{i} p_{i}\left(\lambda_{1}\right) \widetilde{N}_{i}\left(t-\tau_{i}\right) \\
& +\alpha_{i}\left(p_{i}\left(\lambda_{1}+\widetilde{S}\left(t-\tau_{i}\right)\right)-p_{i}\left(\lambda_{1}\right)\right) \widetilde{N}_{i}\left(t-\tau_{i}\right), \quad i=2,3, \ldots, n .
\end{aligned}\right.
$$

Наконец, перепишем систему (2.12) в наиболее компактном виде:

$$
\frac{d}{d t} y(t)=\mathbf{A} y(t)+\sum_{i=1}^{n} \mathbf{B}_{i} y\left(t-\tau_{i}\right)+\mathbf{F}(y(t))+\mathbf{G}\left(y\left(t-\tau_{1}\right), \ldots, y\left(t-\tau_{n}\right)\right),
$$

где

$$
\begin{aligned}
& \mathbf{A}=\left(\begin{array}{ccccc}
-\left(D+\gamma_{1}\right) & -D_{1} / \alpha_{1} & -p_{2}\left(\lambda_{1}\right) & \ldots & -p_{n}\left(\lambda_{1}\right) \\
0 & -D_{1} & 0 & \ldots & 0 \\
0 & 0 & -D_{2} & \ldots & 0 \\
\vdots & \vdots & \vdots & \ddots & \vdots \\
0 & 0 & 0 & \ldots & -D_{n}
\end{array}\right), \quad \mathbf{B}_{1}=\left(\begin{array}{ccccc}
0 & 0 & 0 & \ldots & 0 \\
\alpha_{1} \gamma_{1} & D_{1} & 0 & \ldots & 0 \\
0 & 0 & 0 & \ldots & 0 \\
\vdots & \vdots & \vdots & \ddots & \vdots \\
0 & 0 & 0 & \ldots & 0
\end{array}\right) \\
& \mathbf{B}_{2}=\left(\begin{array}{ccccc}
0 & 0 & 0 & \ldots & 0 \\
0 & 0 & 0 & \ldots & 0 \\
0 & 0 & \alpha_{2} p_{2}\left(\lambda_{1}\right) & \ldots & 0 \\
\vdots & \vdots & \vdots & \ddots & \vdots \\
0 & 0 & 0 & \ldots & 0
\end{array}\right), \quad \ldots, \quad \mathbf{B}_{n}=\left(\begin{array}{ccccc}
0 & 0 & 0 & \ldots & 0 \\
0 & 0 & 0 & \ldots & 0 \\
0 & 0 & 0 & \ldots & 0 \\
\vdots & \vdots & \vdots & \ddots & \vdots \\
0 & 0 & 0 & \ldots & \alpha_{n} p_{n}\left(\lambda_{1}\right)
\end{array}\right) \\
& \mathbf{F}(y(t))=\left(\begin{array}{c}
f_{a}(x(t))+f_{b}(x(t))+u(y(t)) \\
0 \\
0 \\
\vdots \\
0
\end{array}\right) \\
& \mathbf{G}\left(y\left(t-\tau_{1}\right), \ldots, y\left(t-\tau_{n}\right)\right)=\left(\begin{array}{c}
0 \\
\alpha_{1}\left(f_{a}\left(x\left(t-\tau_{1}\right)\right)+f_{b}\left(x\left(t-\tau_{1}\right)\right)\right) \\
\alpha_{2}\left(p_{2}\left(\lambda_{1}+\widetilde{S}\left(t-\tau_{2}\right)\right)-p_{2}\left(\lambda_{1}\right)\right) \widetilde{N}_{2}\left(t-\tau_{2}\right) \\
\vdots \\
\alpha_{n}\left(p_{n}\left(\lambda_{1}+\widetilde{S}\left(t-\tau_{n}\right)\right)-p_{n}\left(\lambda_{1}\right)\right) \widetilde{N}_{n}\left(t-\tau_{n}\right)
\end{array}\right) .
\end{aligned}
$$

Для линеаризованной системы

$$
\frac{d}{d t} y(t)=\mathbf{A} y(t)+\sum_{i=1}^{n} \mathbf{B}_{i} y\left(t-\tau_{i}\right)
$$


характеристический квазимногочлен будет иметь вид:

$$
\operatorname{det}\left(\lambda \mathbf{I}-\mathbf{A}-\sum_{i=1}^{n} e^{-\lambda \tau_{i}} \mathbf{B}_{i}\right)=\operatorname{det}\left(\lambda I-A_{1}-e^{-\lambda \tau_{1}} B_{1}\right) \prod_{i=2}^{n}\left(\lambda+D_{i}-\alpha_{i} p_{i}\left(\lambda_{1}\right) e^{-\lambda \tau_{i}}\right),
$$

где

$$
\begin{aligned}
& \operatorname{det}\left(\lambda I-A_{1}-e^{-\lambda \tau_{1}} B_{1}\right)=\left|\begin{array}{cc}
\lambda+D+\gamma_{1} & D_{1} / \alpha_{1} \\
-\alpha_{1} \gamma_{1} e^{-\lambda \tau_{1}} & \lambda+D_{1}-D_{1} e^{-\lambda \tau_{1}}
\end{array}\right|= \\
& \quad=\left(\lambda+D+\gamma_{1}\right)\left(\lambda+D_{1}-D_{1} e^{-\lambda \tau_{1}}\right)+\gamma_{1} D_{1} e^{-\lambda \tau_{1}}=\left(\lambda+D+\gamma_{1}\right)\left(\lambda+D_{1}\right)-(\lambda+D) D_{1} e^{-\lambda \tau_{1}} .
\end{aligned}
$$

Здесь $\mathbf{I}$ - единичная матрица размера $(n+1) \times(n+1), I$ - единичная матрица размера $2 \times 2$.

Хорошо известно (см., например, [4, гл. $7, \S 33])$, что если все корни характеристического квазимногочлена лежат в левой полуплоскости $\{\lambda \in \mathbb{C}: \operatorname{Re} \lambda<0\}$, то нулевое решение системы $(2.13)$ асимптотически устойчиво. Если же существует хотя бы один корень квазимногочлена, лежащий в правой полуплоскости $\{\lambda \in \mathbb{C}: \operatorname{Re} \lambda>0\}$, то нулевое решение системы (2.13) неустойчиво.

Учитывая, что исследование устойчивости нулевого решения системы (2.13) эквивалентно исследованию устойчивости положения равновесия (1.4) системы (1.1), нетрудно установить следующий результат.

Теорема 1. Пусть $\lambda_{1}<S^{0}$ и функиия $p_{1}(S)$ является непрерывно дифберенцируемой в некоторой окрестности точки $S=\lambda_{1}$.

(а) Если $p_{1}^{\prime}\left(\lambda_{1}\right)>0$ и $p_{i}\left(\lambda_{1}\right)<D_{i} / \alpha_{i}, i=2, \ldots, n$, то положение равновесия (1.4) является асимптотически устойчивым.

(b) Если существует такой номер $i_{0} \in\{2, \ldots, n\}$, что $p_{i_{0}}\left(\lambda_{1}\right)>D_{i_{0}} / \alpha_{i_{0}}$, то положение равновесия (1.4) неустойчиво.

Доказательство. (а) Вначале покажем, что все корни квазимногочлена

$$
Q_{1}(\lambda)=\left(\lambda+D+\gamma_{1}\right)\left(\lambda+D_{1}\right)-(\lambda+D) D_{1} e^{-\lambda \tau_{1}}
$$

содержатся в левой полуплоскости $\{\lambda \in \mathbb{C}: \operatorname{Re} \lambda<0\}$. Предположим, что существует корень квазимногочлена $Q_{1}(\lambda)$ с неотрицательной вещественной частью: $\operatorname{Re} \lambda \geqslant 0$. Тогда

$$
\frac{\left(\lambda+D+\gamma_{1}\right)}{(\lambda+D)}=\frac{D_{1}}{\left(\lambda+D_{1}\right)} e^{-\lambda \tau_{1}}
$$

Поскольку $p_{1}^{\prime}\left(\lambda_{1}\right)>0$, то в силу обозначения $(2.5)$ имеем $\gamma_{1}>0$. Следовательно,

$$
1<\left|\frac{\left(\lambda+D+\gamma_{1}\right)}{(\lambda+D)}\right|=\left|\frac{D_{1}}{\left(\lambda+D_{1}\right)} e^{-\lambda \tau_{1}}\right| \leqslant 1
$$

противоречие. Теперь покажем, что все корни квазимногочленов

$$
Q_{i}(\lambda)=\lambda+D_{i}-\alpha_{i} p_{i}\left(\lambda_{1}\right) e^{-\lambda \tau_{i}}, \quad i=2, \ldots, n,
$$

также содержатся в левой полуплоскости $\{\lambda \in \mathbb{C}: \operatorname{Re} \lambda<0\}$. Также будем рассуждать от противного. Предположим, что существует корень $Q_{i}(\lambda)$ с неотрицательной вещественной частью: $\operatorname{Re} \lambda \geqslant 0$. В этом случае

$$
D_{i} \leqslant\left|\lambda+D_{i}\right|=\left|\alpha_{i} p_{i}\left(\lambda_{1}\right) e^{-\lambda \tau_{i}}\right| \leqslant \alpha_{i} p_{i}\left(\lambda_{1}\right) .
$$

Получаем противоречие с условием теоремы. Итак, положение равновесия (1.4) асимптотически устойчиво.

(b) Рассмотрим функцию

$$
Q_{i_{0}}(\lambda)=\lambda+D_{i_{0}}-\alpha_{i_{0}} p_{i_{0}}\left(\lambda_{1}\right) e^{-\lambda \tau_{i_{0}}}
$$

как функцию вещественного аргумента. По условию теоремы $Q_{i_{0}}(0)=D_{i_{0}}-\alpha_{i_{0}} p_{i_{0}}\left(\lambda_{1}\right)<0$. C другой стороны, $Q_{i_{0}}(\lambda) \rightarrow+\infty$ при $\lambda \rightarrow+\infty$. Поскольку функция $Q_{i_{0}}(\lambda)$ является непрерывной, значит, существует такое число $\lambda^{*}>0$, что $Q_{i_{0}}\left(\lambda^{*}\right)=0$. Итак, положение равновесия (1.4) неустойчиво. Теорема 1 доказана. 
Следующая наша цель - получение оценок, характеризующих скорость стабилизации решений на бесконечности к положению равновесия (1.4) и нахождение оценок на множество притяжения в случае, когда рассматриваемое положение равновесия является асимптотически устойчивым. При получении оценок мы будем использовать модифицированные функционалы ЛяпуноваКрасовского. В следующем разделе мы укажем один из методов построения таких функционалов для системы $(2.13)$ в случае $n=1$.

3. Построение модифицированного функционала Ляпунова-Красовского для случая $n=1$. В данном разделе будем предполагать, что выполнено условие $\lambda_{1}<S^{0}$, функция $p_{1}(S)$ является непрерывно дифференцируемой в некоторой окрестности точки $S=\lambda_{1}$ и $p_{1}^{\prime}\left(\lambda_{1}\right)>0$. Если $n=1$, то в силу теоремы 1 эти условия гарантируют асимптотическую устойчивость нетривиального положения равновесия (1.4) системы (1.1).

Как отмечалось выше, исследование устойчивости положения равновесия (1.4) системы (1.1) эквивалентно исследованию устойчивости нулевого решения системы (2.13). В случае $n=1$ система (2.13) запишется в виде

$$
\frac{d}{d t} x(t)=A_{1} x(t)+B_{1} x\left(t-\tau_{1}\right)+F_{1}(x(t))+G_{1}\left(x\left(t-\tau_{1}\right)\right),
$$

где $x(t), A_{1}, B_{1}, F_{1}(x(t)), G_{1}\left(x\left(t-\tau_{1}\right)\right)$ определены в (2.4)-(2.9).

Одним из методов исследования устойчивости решений систем с запаздыванием является метод функционалов Ляпунова-Красовского, предложенный Н. Н. Красовским (см. [4]). В частности, для линейных систем вида

$$
\frac{d}{d t} y(t)=A y(t)+B y(t-\tau)
$$

где $A$ и $B$ - постоянные матрицы, Н. Н. Красовский предложил использовать функционал

$$
V_{0}(t, y)=\left\langle H_{0} y(t), y(t)\right\rangle+\int_{t-\tau}^{t}\left\langle K_{0} y(s), y(s)\right\rangle d s
$$

с матрицами $H_{0}=H_{0}^{*}>0$ и $K_{0}=K_{0}^{*}>0$ (см. [4, гл. $\left.\left.7, \S 34\right]\right)$. Здесь и далее через $H_{0}^{*}$ обозначается эрмитово-сопряженная к $H_{0}$ матрица, неравенство $H_{0}>0$ означает, что матрица $H_{0}$ является положительно определенной. Отметим, что с помощью функционала (3.3) также можно проводить исследования устойчивости решений и для нелинейных систем с запаздыванием (в частности, для системы вида (3.1)).

Однако, используя функционал (3.3), далеко не всегда удается получить оценки решений, характеризующие скорость убывания на бесконечности. Оценки удается получить с помощью модифицированных функционалов Ляпунова-Красовского (см. $[2,12,13])$. В частности, в [2] был предложен модифицированный функционал Ляпунова-Красовского следующего вида:

$$
V(t, y)=\langle H y(t), y(t)\rangle+\int_{t-\tau}^{t}\langle K(t-s) y(s), y(s)\rangle d s
$$

где матрицы $H$ и $K(s) \in C^{1}([0, \tau])$ обладают следующими свойствами:

$$
H=H^{*}>0, \quad K(s)=K^{*}(s)>0, \quad \frac{d}{d s} K(s)<0, \quad s \in[0, \tau] .
$$

При этом для асимптотической устойчивости нулевого решения системы (3.2) достаточно потребовать выполнение неравенства

$$
C=-\left(\begin{array}{cc}
H A+A^{*} H+K(0) & H B \\
B^{*} H & -K(\tau)
\end{array}\right)>0 .
$$

При выполнении условий (3.5), (3.6) в работе [2] были получены оценки решений системы (3.2), характеризующие скорость убывания на бесконечности. Также в $[2,3,5]$ для некоторых классов нелинейных систем с запаздыванием были установлены оценки решений и указаны оценки на 
области притяжения. Полученные результаты также были обобщены на случай систем с несколькими запаздываниями.

Отметим, что примеры построения модифицированных функционалов Ляпунова-Красовского вида (3.4) были приведены, в частности, в [6-9] при исследовании устойчивости положений равновесия в различных биологических моделях. С помощью построенных функционалов были получены конструктивные оценки решений, характеризующие скорость стабилизации на бесконечности, и указаны оценки множеств притяжения.

Данный раздел посвящен построению модифицированного функционала Ляпунова-Красовского для системы (3.1). Рассмотрим функционал

$$
V(t, x)=\left\langle H_{1} x(t), x(t)\right\rangle+\int_{t-\tau_{1}}^{t}\left\langle K_{1}(t-s) x(s), x(s)\right\rangle d s,
$$

где

$$
H_{1}=\left(\begin{array}{ll}
h_{00} & h_{01} \\
h_{01} & h_{11}
\end{array}\right)>0, \quad K_{1}(s)=e^{-\kappa_{1} s}\left(B_{1}^{*} B_{1}+M_{1}\right), \quad M_{1}=M_{1}^{*}>0, \quad \kappa_{1}>0, \quad s \in\left[0, \tau_{1}\right] .
$$

Подберем матрицы $H_{1}$ и $K_{1}(s)$ так, чтобы было выполнено неравенство

$$
C_{1}=-\left(\begin{array}{cc}
H_{1} A_{1}+A_{1}^{*} H_{1}+K_{1}(0) & H_{1} B_{1} \\
B_{1}^{*} H_{1} & -K_{1}\left(\tau_{1}\right)
\end{array}\right)>0 .
$$

Учитывая явный вид матрицы $B_{1}$, нетрудно заметить, что $B_{1}^{*} H_{1}=B_{1}^{*} \widetilde{H}_{1}$, где

$$
\widetilde{H}_{1}=\left(\begin{array}{cc}
0 & 0 \\
h_{01} & h_{11}
\end{array}\right) \text {. }
$$

Следовательно,

$$
\begin{aligned}
C_{1}=\left(\begin{array}{cc}
-\left(H_{1} A_{1}+A_{1}^{*} H_{1}+B_{1}^{*} B_{1}\right)-M_{1} & -\widetilde{H}_{1}^{*} B_{1} \\
-B_{1}^{*} \widetilde{H}_{1} & e^{-\kappa_{1} \tau_{1}}\left(B_{1}^{*} B_{1}+M_{1}\right)
\end{array}\right)= \\
=\left(\begin{array}{cc}
-\left(H_{1} A_{1}+A_{1}^{*} H_{1}+B_{1}^{*} B_{1}+e^{\kappa_{1} \tau_{1}} \widetilde{H}_{1}^{*} \widetilde{H}_{1}\right)-M_{1} & 0 \\
0 & e^{-\kappa_{1} \tau_{1}} M_{1}
\end{array}\right)+\left(\begin{array}{cc}
e^{\kappa_{1} \tau_{1}} \widetilde{H}_{1}^{*} \widetilde{H}_{1} & -\widetilde{H}_{1}^{*} B_{1} \\
-B_{1}^{*} \widetilde{H}_{1} & e^{-\kappa_{1} \tau_{1}} B_{1}^{*} B_{1}
\end{array}\right)= \\
=\left(\begin{array}{cc}
R_{1}-M_{1} & 0 \\
0 & e^{-\kappa_{1} \tau_{1}} M_{1}
\end{array}\right)+\left(\begin{array}{cc}
e^{\kappa_{1} \tau_{1}} \widetilde{H}_{1}^{*} \widetilde{H}_{1} & -\widetilde{H}_{1}^{*} B_{1} \\
-B_{1}^{*} \widetilde{H}_{1} & e^{-\kappa_{1} \tau_{1}} B_{1}^{*} B_{1}
\end{array}\right),
\end{aligned}
$$

где

$$
R_{1}=-\left(H_{1} A_{1}+A_{1}^{*} H_{1}+B_{1}^{*} B_{1}+e^{\kappa_{1} \tau_{1}} \widetilde{H}_{1}^{*} \widetilde{H}_{1}\right)=\left(\begin{array}{cc}
r_{00} & r_{01} \\
r_{01} & r_{11}
\end{array}\right) .
$$

Из данного представления вытекает неравенство

$$
\left\langle C_{1}\left(\begin{array}{l}
u \\
v
\end{array}\right),\left(\begin{array}{l}
u \\
v
\end{array}\right)\right\rangle \geqslant\left\langle\left(R_{1}-M_{1}\right) u, u\right\rangle+e^{-\kappa_{1} \tau_{1}}\left\langle M_{1} v, v\right\rangle, \quad u, v \in \mathbb{R}^{2} .
$$

Тем самым, если мы покажем, что матрица $R_{1}$ является положительно определенной, то при условии $\left\|M_{1}\right\|<r_{1, \text { min }}$ матрица $C_{1}$ также будет положительно определена. (Здесь $\left\|M_{1}\right\|$ - спектральная норма матрицы $M_{1}, r_{1, \min }>0$ - минимальное собственное значение матрицы $R_{1}$.)

Итак, наша цель - подобрать величины $h_{00}>0, h_{11}>0, h_{01} \in \mathbb{R}\left(h_{00} h_{11}-h_{01}^{2}>0\right)$ и $\kappa_{1}>0$ так, чтобы матрица $R_{1}$ была положительно определенной. Учитывая вид матриц $H_{1}, \widetilde{H}_{1}, A_{1}$ и $B_{1}$, получим

$$
\left\{\begin{array}{l}
r_{00}=2\left(D+\gamma_{1}\right) h_{00}-\alpha_{1}^{2} \gamma_{1}^{2}-e^{\kappa_{1} \tau_{1}} h_{01}^{2}, \\
r_{01}=\frac{D_{1}}{\alpha_{1}} h_{00}+\left(D+\gamma_{1}+D_{1}\right) h_{01}-\alpha_{1} \gamma_{1} D_{1}-e^{\kappa_{1} \tau_{1}} h_{01} h_{11} \\
r_{11}=\frac{2 D_{1}}{\alpha_{1}} h_{01}+2 D_{1} h_{11}-D_{1}^{2}-e^{\kappa_{1} \tau_{1}} h_{11}^{2}
\end{array}\right.
$$


при этом определитель матрицы $R_{1}$ имеет вид

$$
\operatorname{det} R_{1}=r_{11}\left(2\left(D+\gamma_{1}\right) h_{00}-\alpha_{1}^{2} \gamma_{1}^{2}-e^{\kappa_{1} \tau_{1}} h_{01}^{2}\right)--\left(\frac{D_{1}}{\alpha_{1}} h_{00}+\left(D+\gamma_{1}+D_{1}\right) h_{01}-\alpha_{1} \gamma_{1} D_{1}-e^{\kappa_{1} \tau_{1}} h_{01} h_{11}\right)^{2} .
$$

Величину $h_{00}$ выберем из условия, чтобы определитель матрицы $R_{1}$ принимал наибольшее значение. Тогда

$$
h_{00}=\frac{\alpha_{1}}{D_{1}}\left(\frac{\alpha_{1}}{D_{1}}\left(D+\gamma_{1}\right) r_{11}-\left(D+\gamma_{1}+D_{1}\right) h_{01}+\alpha_{1} \gamma_{1} D_{1}+e^{\kappa_{1} \tau_{1}} h_{01} h_{11}\right) .
$$

В этом случае

$$
\operatorname{det} R_{1}=r_{11}\left(\alpha_{1}^{2}\left(e^{-\kappa_{1} \tau_{1}}\left(D+\gamma_{1}\right)^{2}-D^{2}\right)-e^{\kappa_{1} \tau_{1}}\left(h_{01}-\frac{\alpha_{1}}{D_{1}}\left(D+\gamma_{1}\right)\left(h_{11}-e^{-\kappa_{1} \tau_{1}} D_{1}\right)\right)^{2}\right)
$$

Полагая

$$
h_{01}=\frac{\alpha_{1}}{D_{1}}\left(D+\gamma_{1}\right)\left(h_{11}-e^{-\kappa_{1} \tau_{1}} D_{1}\right),
$$

будем иметь

$$
\operatorname{det} R_{1}=r_{11} \alpha_{1}^{2}\left(e^{-\kappa_{1} \tau_{1}}\left(D+\gamma_{1}\right)^{2}-D^{2}\right)
$$

где

$$
r_{11}=2\left(D+\gamma_{1}+D_{1}\right) h_{11}-2 e^{-\kappa_{1} \tau_{1}}\left(D+\gamma_{1}\right) D_{1}-D_{1}^{2}-e^{\kappa_{1} \tau_{1}} h_{11}^{2} .
$$

Число $h_{11}$ выберем так, чтобы величина $r_{11}$ принимала наибольшее значение. Положим

$$
h_{11}=e^{-\kappa_{1} \tau_{1}}\left(D+\gamma_{1}+D_{1}\right)
$$

тогда

$$
r_{11}=e^{-\kappa_{1} \tau_{1}}\left(\left(D+\gamma_{1}\right)^{2}+D_{1}^{2}\right)-D_{1}^{2} .
$$

Итак, окончательно получим

$$
\begin{aligned}
& \left\{\begin{array}{l}
h_{00}=\frac{\alpha_{1}^{2}}{D_{1}^{2}}\left(e^{-\kappa_{1} \tau_{1}}\left(D+\gamma_{1}\right)\left(\left(D+\gamma_{1}\right)^{2}+D_{1}^{2}\right)-D D_{1}^{2}\right) \\
h_{01}=e^{-\kappa_{1} \tau_{1}} \frac{\alpha_{1}}{D_{1}}\left(D+\gamma_{1}\right)^{2} \\
h_{11}=e^{-\kappa_{1} \tau_{1}}\left(D+\gamma_{1}+D_{1}\right)
\end{array}\right. \\
& \left\{\begin{array}{l}
r_{00}=\frac{\alpha_{1}^{2}}{D_{1}^{2}}\left(D+\gamma_{1}\right)^{2}\left(e^{-\kappa_{1} \tau_{1}}\left(\left(D+\gamma_{1}\right)^{2}+D_{1}^{2}\right)-D_{1}^{2}\right)+\alpha_{1}^{2}\left(e^{-\kappa_{1} \tau_{1}}\left(D+\gamma_{1}\right)^{2}-D^{2}\right), \\
r_{01}=\frac{\alpha_{1}}{D_{1}}\left(D+\gamma_{1}\right)\left(e^{-\kappa_{1} \tau_{1}}\left(\left(D+\gamma_{1}\right)^{2}+D_{1}^{2}\right)-D_{1}^{2}\right), \\
r_{11}=e^{-\kappa_{1} \tau_{1}}\left(\left(D+\gamma_{1}\right)^{2}+D_{1}^{2}\right)-D_{1}^{2},
\end{array}\right.
\end{aligned}
$$

причем

$$
\operatorname{det} R_{1}=\alpha_{1}^{2}\left(e^{-\kappa_{1} \tau_{1}}\left(\left(D+\gamma_{1}\right)^{2}+D_{1}^{2}\right)-D_{1}^{2}\right)\left(e^{-\kappa_{1} \tau_{1}}\left(D+\gamma_{1}\right)^{2}-D^{2}\right) .
$$

Согласно критерию Сильвестра для положительной определенности матрицы $R_{1}$ необходимо и достаточно выполнения неравенств $r_{11}>0$ и $\operatorname{det} R_{1}>0$. В нашем случае эти неравенства запишутся в виде

$$
e^{\kappa_{1} \tau_{1}}<1+\frac{\left(D+\gamma_{1}\right)^{2}}{D_{1}^{2}}, \quad e^{\kappa_{1} \tau_{1}}<\frac{\left(D+\gamma_{1}\right)^{2}}{D^{2}} .
$$

Из формулы $(2.5)$ и условия $p_{1}^{\prime}\left(\lambda_{1}\right)>0$ следует, что $\gamma_{1}>0$, поэтому всегда можно подобрать такое $\kappa_{1}>0$, что неравенства (3.12) будут выполнены.

Отметим, что в случае, когда величина $\kappa_{1}>0$ определяется из неравенств $(3.12)$, матрица $H_{1}$ является положительно определенной. Действительно, из (3.8) вытекает, что

$$
H_{1} A_{1}+A_{1}^{*} H_{1}=-B_{1}^{*} B_{1}-e^{\kappa_{1} \tau_{1}} \widetilde{H}_{1}^{*} \widetilde{H}_{1}-R_{1}<0,
$$


т.е. матрица $H_{1}=H_{1}^{*}$ является решением матричного уравнения Ляпунова

$$
H_{1} A_{1}+A_{1}^{*} H_{1}=-S_{1},
$$

где $S_{1}=S_{1}^{*}>0$. Поскольку все собственные значения матрицы $A_{1}$ содержатся в левой полуплоскости $\{\lambda \in \mathbb{C}: \operatorname{Re} \lambda<0\}$, отсюда следует положительная определенность матрицы $H_{1}$ (см., например, $[1$, гл. $1, \S 4])$. Итак, модифицированный функционал Ляпунова-Красовского построен.

4. Оценки скорости сходимости к нетривиальному положению равновесия в случае $n=1$. В данном разделе будем предполагать, что выполнено условие $\lambda_{1}<S^{0}$, функция $p_{1}(S)$ является непрерывно дифференцируемой в некоторой окрестности точки $S=\lambda_{1}$ и $p_{1}^{\prime}\left(\lambda_{1}\right)>0$. При $n=1$ эти условия гарантируют асимптотическую устойчивость нетривиального положения равновесия (1.4) системы (1.1).

Рассмотрим начальную задачу для системы (3.1):

$$
\left\{\begin{array}{l}
\frac{d}{d t} x(t)=A_{1} x(t)+B_{1} x\left(t-\tau_{1}\right)+F_{1}(x(t))+G_{1}\left(x\left(t-\tau_{1}\right)\right), \\
x(t)=\psi(t), \quad t \in\left[-\tau_{1}, 0\right], \quad x(+0)=\psi(0)
\end{array}\right.
$$

где $x(t), A_{1}, B_{1}, F_{1}(x(t)), G_{1}\left(x\left(t-\tau_{1}\right)\right)$ определены в $(2.4)-(2.9)$,

$$
\psi(t)=\left(\begin{array}{l}
\psi_{0}(t) \\
\psi_{1}(t)
\end{array}\right), \quad\left\{\begin{array}{l}
\psi_{0}(t)=\varphi_{0}(t)-\lambda_{1} \\
\psi_{1}(t)=\varphi_{1}(t)-\frac{\alpha_{1}}{D_{1}}\left(S^{0}-\lambda_{1}\right) D,
\end{array}\right.
$$

$\varphi_{0}(t), \varphi_{1}(t)$ - непрерывные неотрицательные функции, заданные в (1.2).

Наша цель - получение оценок решений начальной задачи (4.1), характеризующих скорость убывания на бесконечности, и нахождение множества притяжения нулевого решения. В силу замены (2.1) из полученных оценок будут вытекать соответствующие результаты для начальной задачи (1.1)-(1.2) при $n=1$.

Оценки будут получены с использованием модифицированного функционала ЛяпуноваКрасовского, построенного в предыдущем разделе:

$$
V(t, x)=\left\langle H_{1} x(t), x(t)\right\rangle+\int_{t-\tau_{1}}^{t}\left\langle K_{1}(t-s) x(s), x(s)\right\rangle d s,
$$

где

$$
H_{1}=\left(\begin{array}{ll}
h_{00} & h_{01} \\
h_{01} & h_{11}
\end{array}\right)>0, \quad K_{1}(s)=e^{-\kappa_{1} s}\left(B_{1}^{*} B_{1}+M_{1}\right), \quad M_{1}=M_{1}^{*}>0, \quad \kappa_{1}>0, \quad s \in\left[0, \tau_{1}\right],
$$

величины $h_{00}, h_{01}, h_{11}$ определены в (3.10), число $\kappa_{1}$ определено в (3.12), матрица $M_{1}$ будет определена ниже.

Введем следующие обозначения. Пусть $c_{1}>0$-такое число, что выполнено неравенство

$$
\left\langle R_{1} u, u\right\rangle \geqslant c_{1}\left\langle H_{1} u, u\right\rangle, \quad u \in \mathbb{R}^{2},
$$

где матрица $R_{1}>0$ определена в (3.8), (3.11). Например, можно взять $c_{1}=r_{1, \min } /\left\|H_{1}\right\|$, где

$$
r_{1, \min }=\frac{1}{2}\left(r_{00}+r_{11}\right)-\sqrt{\frac{1}{4}\left(r_{00}-r_{11}\right)^{2}+r_{01}^{2}}, \quad\left\|H_{1}\right\|=\frac{1}{2}\left(h_{00}+h_{11}\right)+\sqrt{\frac{1}{4}\left(h_{00}-h_{11}\right)^{2}+h_{01}^{2}} .
$$

Наибольшее число $c_{1}>0$, при котором выполняется неравенство (4.3), имеет вид

$$
\begin{aligned}
c_{1}=\frac{1}{\left(h_{00} h_{11}-h_{01}^{2}\right)}\left(\frac{1}{2}\left(h_{00} r_{11}+h_{11} r_{00}-2 h_{01} r_{01}\right)-\right. \\
\left.\quad-\sqrt{\left(\frac{1}{2}\left(h_{00} r_{11}-h_{11} r_{00}-2 h_{01} r_{01}\right)+h_{11} \frac{r_{01}^{2}}{r_{11}}\right)^{2}+\left(h_{11} \frac{r_{01}}{r_{11}}-h_{01}\right)^{2}\left(r_{00} r_{11}-r_{01}^{2}\right)}\right) .
\end{aligned}
$$


Предположим, что функция $p_{1}(S)$ является дважды непрерывно дифференцируемой. При $\theta>0$ рассмотрим функции

$$
\zeta_{1}(\theta)=\max _{\substack{|s| \leqslant \theta \\ \lambda_{1}+s \geqslant 0}}\left|p_{1}^{\prime \prime}\left(\lambda_{1}+s\right)\right|, \quad \eta_{1}(\theta)=\max _{\substack{|s| \leqslant \theta \\ \lambda_{1}+s \geqslant 0}}\left|p_{1}^{\prime}\left(\lambda_{1}+s\right)\right| .
$$

Пусть $\theta>0$ таково, что выполнено неравенство

$$
2 e^{\kappa_{1} \tau_{1} / 2} \sqrt{h_{11} \nu_{1}} \alpha_{1} \theta<c_{1},
$$

где

Положим

$$
\nu_{1}=\frac{h_{00} \eta_{1}^{2}(\theta)+h_{01} a_{1} \zeta_{1}(\theta) \eta_{1}(\theta)+\frac{1}{4} h_{11} a_{1}^{2} \zeta_{1}^{2}(\theta)}{h_{00} h_{11}-h_{01}^{2}}, \quad a_{1}=\frac{\alpha_{1}}{D_{1}}\left(S^{0}-\lambda_{1}\right) D
$$

$$
M_{1}=\mu_{1} H_{1}, \quad \mu_{1}=e^{\kappa_{1} \tau_{1} / 2} \sqrt{h_{11} \nu_{1}} \alpha_{1} \theta .
$$

Также введем обозначения

$$
\begin{gathered}
\delta_{1}=\min \left\{\left(c_{1}-2 e^{\kappa_{1} \tau_{1} / 2} \sqrt{h_{11} \nu_{1}} \alpha_{1} \theta\right), \kappa_{1}\right\}>0, \quad q_{1}=\sqrt{h_{00}} \xi_{1} \\
\xi_{1}=\frac{h_{01} \eta_{1}(\theta)+\frac{1}{2} h_{11} a_{1} \zeta_{1}(\theta)+\sqrt{h_{11}\left(h_{00} \eta_{1}^{2}(\theta)+h_{01} a_{1} \zeta_{1}(\theta) \eta_{1}(\theta)+\frac{1}{4} h_{11} a_{1}^{2} \zeta_{1}^{2}(\theta)\right)}}{h_{00} h_{11}-h_{01}^{2}} \\
s_{1}=\frac{h_{11}}{\left(h_{00} h_{11}-h_{01}^{2}\right)}, \quad n_{1}=\frac{h_{00}}{\left(h_{00} h_{11}-h_{01}^{2}\right)} .
\end{gathered}
$$

Сформулируем основные результаты настоящего параграфа.

Теорема 2. Пусть $\lambda_{1}<S^{0}$, функиия $p_{1}(S)$ является дважды непрерывно дифферениируемой и $p_{1}^{\prime}\left(\lambda_{1}\right)>0$. Тогда для решения $x(t)=\left(\widetilde{S}(t), \widetilde{N}_{1}(t)\right)^{T}$ начальной задачи (4.1) с начальными данными, удовлетворяющими условиям

$$
\begin{gathered}
\max _{t \in\left[-\tau_{1}, 0\right]}\left|\psi_{0}(t)\right| \leqslant \theta, \quad\left|\psi_{0}(0)\right|<\theta, \quad \psi_{0}(t)+\lambda_{1} \geqslant 0, \quad \psi_{1}(t)+\frac{\alpha_{1}}{D_{1}}\left(S^{0}-\lambda_{1}\right) D \geqslant 0, \\
\sqrt{V(0, \psi)}<\frac{\delta_{1}}{q_{1}}, \quad \frac{\sqrt{s_{1}} \sqrt{V(0, \psi)}}{\left(1-\frac{q_{1}}{\delta_{1}} \sqrt{V(0, \psi)}\right)} \leqslant \theta
\end{gathered}
$$

справедливы оценки

$$
\begin{gathered}
|\widetilde{S}(t)| \leqslant \frac{\sqrt{s_{1}} \sqrt{V(0, \psi)}}{\left(1-\frac{q_{1}}{\delta_{1}} \sqrt{V(0, \psi)}\right)} e^{-\delta_{1} t / 2}, \quad t>0, \\
\left|\widetilde{N}_{1}(t)\right| \leqslant \frac{\sqrt{n_{1}} \sqrt{V(0, \psi)}}{\left(1-\frac{q_{1}}{\delta_{1}} \sqrt{V(0, \psi)}\right)} e^{-\delta_{1} t / 2}, \quad t>0 .
\end{gathered}
$$

Доказательство теоремы 2 будет приведено в следующем разделе.

Сформулируем результат для системы (1.1), непосредственно вытекающий из теоремы 2.

Рассмотрим начальную задачу для системы (1.1) при $n=1$ :

$$
\left\{\begin{array}{l}
\frac{d}{d t} S(t)=\left(S^{0}-S(t)\right) D-p_{1}(S(t)) N_{1}(t), \\
\frac{d}{d t} N_{1}(t)=-D_{1} N_{1}(t)+\alpha_{1} p_{1}\left(S\left(t-\tau_{1}\right)\right) N_{1}\left(t-\tau_{1}\right), \\
S(t)=\varphi_{0}(t), \quad t \in\left[-\tau_{1}, 0\right], \quad S(+0)=\varphi_{0}(0), \\
N_{1}(t)=\varphi_{1}(t), \quad t \in\left[-\tau_{1}, 0\right], \quad N_{1}(+0)=\varphi_{1}(0),
\end{array}\right.
$$

где $\varphi_{0}(t), \varphi_{1}(t)$ - непрерывные неотрицательные функции. 
Введем обозначения

Справедлива следующая теорема.

$$
\varphi(t)=\left(\begin{array}{l}
\varphi_{0}(t) \\
\varphi_{1}(t)
\end{array}\right), \quad x^{*}=\left(\begin{array}{c}
\lambda_{1} \\
\frac{\alpha_{1}}{D_{1}}\left(S^{0}-\lambda_{1}\right) D
\end{array}\right) .
$$

Теорема 3. Пусть $\lambda_{1}<S^{0}$, функиия $p_{1}(S)$ является дважды непрерывно дифферениируемой и $p_{1}^{\prime}\left(\lambda_{1}\right)>0$. Тогда для решения $\left(S(t), N_{1}(t)\right)^{T}$ начальной задачи (4.15) с начальными данными, удовлетворяющими условиям

$$
\begin{gathered}
\max _{t \in\left[-\tau_{1}, 0\right]}\left|\varphi_{0}(t)-\lambda_{1}\right| \leqslant \theta, \quad\left|\varphi_{0}(0)-\lambda_{1}\right|<\theta, \quad \varphi_{0}(t) \geqslant 0, \quad \varphi_{1}(t) \geqslant 0, \\
\sqrt{V\left(0, \varphi-x^{*}\right)}<\frac{\delta_{1}}{q_{1}}, \quad \frac{\sqrt{s_{1}} \sqrt{V\left(0, \varphi-x^{*}\right)}}{\left(1-\frac{q_{1}}{\delta_{1}} \sqrt{V\left(0, \varphi-x^{*}\right)}\right)} \leqslant \theta,
\end{gathered}
$$

справедливы оченки

$$
\begin{array}{r}
\left|S(t)-\lambda_{1}\right| \leqslant \frac{\sqrt{s_{1}} \sqrt{V\left(0, \varphi-x^{*}\right)}}{\left(1-\frac{q_{1}}{\delta_{1}} \sqrt{V\left(0, \varphi-x^{*}\right)}\right)} e^{-\delta_{1} t / 2}, \quad t>0, \\
\left|N_{1}(t)-\frac{\alpha_{1}}{D_{1}}\left(S^{0}-\lambda_{1}\right) D\right| \leqslant \frac{\sqrt{n_{1}} \sqrt{V\left(0, \varphi-x^{*}\right)}}{\left(1-\frac{q_{1}}{\delta_{1}} \sqrt{V\left(0, \varphi-x^{*}\right)}\right)} e^{-\delta_{1} t / 2}, \quad t>0 .
\end{array}
$$

5. Доказательство теоремы 2. Рассмотрим модифицированный функционал ЛяпуноваКрасовского (4.2). Дифференцируя его вдоль решения начальной задачи (4.1), получим

$$
\begin{aligned}
& \frac{d}{d t} V(t, x)=\left\langle H_{1} \frac{d}{d t} x(t), x(t)\right\rangle+\left\langle H_{1} x(t), \frac{d}{d t} x(t)\right\rangle+ \\
& +\left\langle K_{1}(0) x(t), x(t)\right\rangle-\left\langle K_{1}\left(\tau_{1}\right) x\left(t-\tau_{1}\right), x\left(t-\tau_{1}\right)\right\rangle+\int_{t-\tau_{1}}^{t}\left\langle\frac{d}{d t} K_{1}(t-s) x(s), x(s)\right\rangle d s= \\
& =\left\langle\left(\begin{array}{cc}
H_{1} A_{1}+A_{1}^{*} H_{1}+K_{1}(0) & H_{1} B_{1} \\
B_{1}^{*} H_{1} & -K_{1}\left(\tau_{1}\right)
\end{array}\right)\left(\begin{array}{c}
x(t) \\
x\left(t-\tau_{1}\right)
\end{array}\right),\left(\begin{array}{c}
x(t) \\
x\left(t-\tau_{1}\right)
\end{array}\right)\right\rangle+ \\
& +2\left\langle H_{1} x(t), F_{1}(x(t))\right\rangle+2\left\langle H_{1} x(t), G_{1}\left(x\left(t-\tau_{1}\right)\right)\right\rangle-\kappa_{1} \int_{t-\tau_{1}}^{t}\left\langle K_{1}(t-s) x(s), x(s)\right\rangle d s .
\end{aligned}
$$

Учитывая обозначение (3.7) и неравенства (3.9) и (4.3), получим оценку

$$
\begin{aligned}
& \left\langle\left(\begin{array}{cc}
H_{1} A_{1}+A_{1}^{*} H_{1}+K_{1}(0) & H_{1} B_{1} \\
B_{1}^{*} H_{1} & -K_{1}\left(\tau_{1}\right)
\end{array}\right)\left(\begin{array}{c}
x(t) \\
x\left(t-\tau_{1}\right)
\end{array}\right),\left(\begin{array}{c}
x(t) \\
x\left(t-\tau_{1}\right)
\end{array}\right)\right\rangle \leqslant \\
& \leqslant-c_{1}\left\langle H_{1} x(t), x(t)\right\rangle+\left\langle M_{1} x(t), x(t)\right\rangle-e^{-\kappa_{1} \tau_{1}}\left\langle M_{1} x\left(t-\tau_{1}\right), x\left(t-\tau_{1}\right)\right\rangle .
\end{aligned}
$$

Отсюда вытекает неравенство

$$
\begin{aligned}
\frac{d}{d t} V(t, x) & \leqslant-c_{1}\left\langle H_{1} x(t), x(t)\right\rangle+\left\langle M_{1} x(t), x(t)\right\rangle-e^{-\kappa_{1} \tau_{1}}\left\langle M_{1} x\left(t-\tau_{1}\right), x\left(t-\tau_{1}\right)\right\rangle \\
+ & 2\left\langle H_{1} x(t), F_{1}(x(t))\right\rangle+2\left\langle H_{1} x(t), G_{1}\left(x\left(t-\tau_{1}\right)\right)\right\rangle-\kappa_{1} \int_{t-\tau_{1}}^{t}\left\langle K_{1}(t-s) x(s), x(s)\right\rangle d s .
\end{aligned}
$$

Вначале предположим, что $t \in\left[0, \tau_{1}\right]$. Оценим

$$
m(t)=\left\langle M_{1} x(t), x(t)\right\rangle+2\left\langle H_{1} x(t), G_{1}\left(x\left(t-\tau_{1}\right)\right)\right\rangle-e^{-\kappa_{1} \tau_{1}}\left\langle M_{1} x\left(t-\tau_{1}\right), x\left(t-\tau_{1}\right)\right\rangle .
$$


Используя явный вид (2.7) вектор-функции $G_{1}\left(x\left(t-\tau_{1}\right)\right)$, будем иметь

$$
\begin{aligned}
& 2\left\langle H_{1} x(t), G_{1}\left(x\left(t-\tau_{1}\right)\right)\right\rangle=2\left\langle H_{1}^{1 / 2} x(t), H_{1}^{1 / 2} G_{1}\left(x\left(t-\tau_{1}\right)\right)\right\rangle \leqslant \\
& \leqslant 2\left\|H_{1}^{1 / 2} x(t)\right\|\left\|H_{1}^{1 / 2} G_{1}\left(x\left(t-\tau_{1}\right)\right)\right\|=2 \sqrt{\left\langle H_{1} x(t), x(t)\right\rangle} \sqrt{h_{11}} \alpha_{1}\left|f_{a}\left(x\left(t-\tau_{1}\right)\right)+f_{b}\left(x\left(t-\tau_{1}\right)\right)\right|,
\end{aligned}
$$

где

$$
\begin{gathered}
f_{a}\left(x\left(t-\tau_{1}\right)\right)=\frac{\alpha_{1}}{D_{1}}\left(S^{0}-\lambda_{1}\right) D\left(p_{1}\left(\lambda_{1}+\widetilde{S}\left(t-\tau_{1}\right)\right)-p_{1}\left(\lambda_{1}\right)-p_{1}^{\prime}\left(\lambda_{1}\right) \widetilde{S}\left(t-\tau_{1}\right)\right), \\
f_{b}\left(x\left(t-\tau_{1}\right)\right)=\left(p_{1}\left(\lambda_{1}+\widetilde{S}\left(t-\tau_{1}\right)\right)-p_{1}\left(\lambda_{1}\right)\right) \widetilde{N}_{1}\left(t-\tau_{1}\right) .
\end{gathered}
$$

Поскольку $t \in\left[0, \tau_{1}\right]$, то $t-\tau_{1} \in\left[-\tau_{1}, 0\right]$. Следовательно, в силу условия (4.11) имеем

$$
\left|\widetilde{S}\left(t-\tau_{1}\right)\right|=\left|\psi_{0}\left(t-\tau_{1}\right)\right| \leqslant \theta .
$$

Учитывая обозначения (4.4), получим следующие оценки:

$$
\begin{gathered}
\left|p_{1}\left(\lambda_{1}+\widetilde{S}\left(t-\tau_{1}\right)\right)-p_{1}\left(\lambda_{1}\right)-p_{1}^{\prime}\left(\lambda_{1}\right) \widetilde{S}\left(t-\tau_{1}\right)\right| \leqslant \frac{1}{2} \zeta_{1}(\theta) \widetilde{S}^{2}\left(t-\tau_{1}\right) \leqslant \frac{1}{2} \zeta_{1}(\theta) \theta\left|\widetilde{S}\left(t-\tau_{1}\right)\right| \\
\left|p_{1}\left(\lambda_{1}+\widetilde{S}\left(t-\tau_{1}\right)\right)-p_{1}\left(\lambda_{1}\right)\right| \leqslant \eta_{1}(\theta)\left|\widetilde{S}\left(t-\tau_{1}\right)\right| \leqslant \eta_{1}(\theta) \theta .
\end{gathered}
$$

Следовательно,

$$
\begin{aligned}
& 2\left\langle H_{1} x(t), G_{1}\left(x\left(t-\tau_{1}\right)\right)\right\rangle \leqslant 2 \sqrt{\left\langle H_{1} x(t), x(t)\right\rangle} \sqrt{h_{11}} \alpha_{1} \theta \times \\
& \times\left(\frac{\alpha_{1}}{2 D_{1}}\left(S^{0}-\lambda_{1}\right) D \zeta_{1}(\theta)\left|\widetilde{S}\left(t-\tau_{1}\right)\right|+\eta_{1}(\theta)\left|\widetilde{N}_{1}\left(t-\tau_{1}\right)\right|\right)= \\
& =2 \sqrt{\left\langle H_{1} x(t), x(t)\right\rangle} \sqrt{h_{11}} \alpha_{1} \theta \sqrt{\left\langle Q_{1}\left(\begin{array}{c}
\left|\widetilde{S}\left(t-\tau_{1}\right)\right| \\
\left|\widetilde{N}_{1}\left(t-\tau_{1}\right)\right|
\end{array}\right),\left(\begin{array}{c}
\left|\widetilde{S}\left(t-\tau_{1}\right)\right| \\
\left|\widetilde{N}_{1}\left(t-\tau_{1}\right)\right|
\end{array}\right)\right\rangle}
\end{aligned}
$$

где

$$
Q_{1}=\left(\begin{array}{cc}
\frac{1}{4} a_{1}^{2} \zeta_{1}^{2}(\theta) & \frac{1}{2} a_{1} \zeta_{1}(\theta) \eta_{1}(\theta) \\
\frac{1}{2} a_{1} \zeta_{1}(\theta) \eta_{1}(\theta) & \eta_{1}^{2}(\theta)
\end{array}\right), \quad a_{1}=\frac{\alpha_{1}}{D_{1}}\left(S^{0}-\lambda_{1}\right) D
$$

Для дальнейших оценок нам понадобится следующее утверждение.

Лемма 1. Пусть $Q$-симметричная матрица с неотрицательными элементами и $H-$ симметричная положительно определенная матрица:

$$
Q=\left(\begin{array}{ll}
q_{00} & q_{01} \\
q_{01} & q_{11}
\end{array}\right), \quad H=\left(\begin{array}{ll}
h_{00} & h_{01} \\
h_{01} & h_{11}
\end{array}\right) .
$$

Тогда для любых $x_{0}, x_{1} \in \mathbb{R}$ справедливо неравенство

$$
\left\langle Q\left(\begin{array}{l}
\left|x_{0}\right| \\
\left|x_{1}\right|
\end{array}\right),\left(\begin{array}{l}
\left|x_{0}\right| \\
\left|x_{1}\right|
\end{array}\right)\right\rangle \leqslant \nu\left\langle H\left(\begin{array}{c}
x_{0} \\
x_{1}
\end{array}\right),\left(\begin{array}{c}
x_{0} \\
x_{1}
\end{array}\right)\right\rangle
$$

где

$$
\begin{aligned}
\nu=\frac{1}{\left(h_{00} h_{11}-h_{01}^{2}\right)}\left(\frac { 1 } { 2 } \left(h_{00} q_{11}\right.\right. & \left.+h_{11} q_{00}+2\left|h_{01}\right| q_{01}\right)+ \\
& \left.+\sqrt{\frac{1}{4}\left(h_{00} q_{11}-h_{11} q_{00}\right)^{2}+\left(h_{00} q_{01}+\left|h_{01}\right| q_{00}\right)\left(h_{11} q_{01}+\left|h_{01}\right| q_{11}\right)}\right) .
\end{aligned}
$$

Доказательство. Неравенство (5.6) можно переписать в виде

$$
\left\langle\left(\begin{array}{cc}
\left(\nu h_{00}-q_{00}\right) & -\left(\nu\left|h_{01}\right|+q_{01}\right) \\
-\left(\nu\left|h_{01}\right|+q_{01}\right) & \left(\nu h_{11}-q_{11}\right)
\end{array}\right)\left(\begin{array}{l}
\left|x_{0}\right| \\
\left|x_{1}\right|
\end{array}\right),\left(\begin{array}{l}
\left|x_{0}\right| \\
\left|x_{1}\right|
\end{array}\right)\right\rangle \geqslant-2 \nu\left(\left|h_{01}\right|\left|x_{0}\right|\left|x_{1}\right|+h_{01} x_{0} x_{1}\right) .
$$


Для справедливости данной оценки достаточно потребовать, чтобы матрица

$$
\left(\begin{array}{cc}
\left(\nu h_{00}-q_{00}\right) & -\left(\nu\left|h_{01}\right|+q_{01}\right) \\
-\left(\nu\left|h_{01}\right|+q_{01}\right) & \left(\nu h_{11}-q_{11}\right)
\end{array}\right)
$$

являлась неотрицательно определенной. Это требование эквивалентно условиям

$$
\nu h_{00}-q_{00} \geqslant 0, \quad \nu h_{11}-q_{11} \geqslant 0, \quad\left(\nu h_{00}-q_{00}\right)\left(\nu h_{11}-q_{11}\right)-\left(\nu\left|h_{01}\right|+q_{01}\right)^{2} \geqslant 0 .
$$

Проводя несложные арифметические выкладки, нетрудно проверить, что эти неравенства будут выполнены. Лемма 1 доказана.

Учитывая обозначение (5.5) для матрицы $Q_{1}$ и используя лемму 1 , получим оценку

$$
\left\langle Q_{1}\left(\begin{array}{c}
\left|\widetilde{S}\left(t-\tau_{1}\right)\right| \\
\left|\widetilde{N}_{1}\left(t-\tau_{1}\right)\right|
\end{array}\right),\left(\begin{array}{c}
\left|\widetilde{S}\left(t-\tau_{1}\right)\right| \\
\left|\widetilde{N}_{1}\left(t-\tau_{1}\right)\right|
\end{array}\right)\right\rangle \leqslant \nu_{1}\left\langle H_{1}\left(\begin{array}{c}
\widetilde{S}\left(t-\tau_{1}\right) \\
\widetilde{N}_{1}\left(t-\tau_{1}\right)
\end{array}\right),\left(\begin{array}{c}
\widetilde{S}\left(t-\tau_{1}\right) \\
\widetilde{N}_{1}\left(t-\tau_{1}\right)
\end{array}\right)\right\rangle,
$$

где $\nu_{1}$ определяется по формуле (4.6). Следовательно,

onyyan

$$
2\left\langle H_{1} x(t), G_{1}\left(x\left(t-\tau_{1}\right)\right)\right\rangle \leqslant 2 \sqrt{\left\langle H_{1} x(t), x(t)\right\rangle} \sqrt{h_{11}} \alpha_{1} \theta \sqrt{\nu_{1}\left\langle H_{1} x\left(t-\tau_{1}\right), x\left(t-\tau_{1}\right)\right\rangle},
$$

$$
\begin{aligned}
m(t) \leqslant\left\langle M_{1} x(t), x(t)\right\rangle-e^{-\kappa_{1} \tau_{1}}\left\langle M_{1} x(\right. & \left.\left.t-\tau_{1}\right), x\left(t-\tau_{1}\right)\right\rangle+ \\
& +2 \sqrt{\left\langle H_{1} x(t), x(t)\right\rangle} \sqrt{h_{11}} \alpha_{1} \theta \sqrt{\nu_{1}\left\langle H_{1} x\left(t-\tau_{1}\right), x\left(t-\tau_{1}\right)\right\rangle} .
\end{aligned}
$$

С учетом обозначения (4.7) нетрудно получить оценку

$$
m(t) \leqslant 2 e^{\kappa_{1} \tau_{1} / 2} \sqrt{h_{11} \nu_{1}} \alpha_{1} \theta\left\langle H_{1} x(t), x(t)\right\rangle .
$$

В силу полученного неравенства при $t \in\left[0, \tau_{1}\right]$ из (5.1) и (5.2) вытекает оценка

$$
\begin{aligned}
& \frac{d}{d t} V(t, x) \leqslant-\left(c_{1}-2 e^{\kappa_{1} \tau_{1} / 2} \sqrt{h_{11} \nu_{1}} \alpha_{1} \theta\right)\left\langle H_{1} x(t), x(t)\right\rangle+ \\
& +2\left\langle H_{1} x(t), F_{1}(x(t))\right\rangle-\kappa_{1} \int_{t-\tau_{1}}^{t}\left\langle K_{1}(t-s) x(s), x(s)\right\rangle d s .
\end{aligned}
$$

Теперь оценим $2\left\langle H_{1} x(t), F_{1}(x(t))\right\rangle$. Учитывая явный вид $(2.6)$ вектор-функции $F_{1}(x(t))$, получим оценку

$$
\begin{aligned}
2\left\langle H_{1} x(t), F_{1}(x(t))\right\rangle=2\left\langle H_{1}^{1 / 2} x(t), H_{1}^{1 / 2} F_{1}(x(t))\right\rangle & \leqslant \\
\leqslant 2\left\|H_{1}^{1 / 2} x(t)\right\|\left\|H_{1}^{1 / 2} F_{1}(x(t))\right\| & =2 \sqrt{\left\langle H_{1} x(t), x(t)\right\rangle} \sqrt{h_{00}}\left(\left|f_{a}(x(t))+f_{b}(x(t))\right|\right),
\end{aligned}
$$

где

$$
\begin{aligned}
f_{a}(x(t))= & \frac{\alpha_{1}}{D_{1}}\left(S^{0}-\lambda_{1}\right) D\left(p_{1}\left(\lambda_{1}+\widetilde{S}(t)\right)-p_{1}\left(\lambda_{1}\right)-p_{1}^{\prime}\left(\lambda_{1}\right) \widetilde{S}(t)\right) \\
& f_{b}(x(t))=\left(p_{1}\left(\lambda_{1}+\widetilde{S}(t)\right)-p_{1}\left(\lambda_{1}\right)\right) \widetilde{N}_{1}(t) .
\end{aligned}
$$

В силу условия (4.11) имеем $|\widetilde{S}(0)|=\left|\psi_{0}(0)\right|<\theta$. Покажем, что неравенство $|\widetilde{S}(t)|<\theta$ также будет выполнено при $t \in\left[0, \tau_{1}\right]$. Предположим, напротив, что существует такая точка $t^{*} \in\left(0, \tau_{1}\right]$, что $|\widetilde{S}(t)|<\theta$ при $t \in\left[0, t^{*}\right)$ и $\left|\widetilde{S}\left(t^{*}\right)\right|=\theta$. Тогда при $t \in\left[0, t^{*}\right]$ по аналогии с (5.3), (5.4) установим справедливость неравенств

Отсюда следует оценка

$$
\begin{gathered}
\left|p_{1}\left(\lambda_{1}+\widetilde{S}(t)\right)-p_{1}\left(\lambda_{1}\right)-p_{1}^{\prime}\left(\lambda_{1}\right) \widetilde{S}(t)\right| \leqslant \frac{1}{2} \zeta_{1}(\theta) \widetilde{S}^{2}(t), \\
\left|p_{1}\left(\lambda_{1}+\widetilde{S}(t)\right)-p_{1}\left(\lambda_{1}\right)\right| \leqslant \eta_{1}(\theta)|\widetilde{S}(t)| .
\end{gathered}
$$

$$
2\left\langle H_{1} x(t), F_{1}(x(t))\right\rangle \leqslant \sqrt{\left\langle H_{1} x(t), x(t)\right\rangle} \sqrt{h_{00}} \times
$$




$$
\begin{gathered}
\times\left(\frac{\alpha_{1}}{D_{1}}\left(S^{0}-\lambda_{1}\right) D \zeta_{1}(\theta) \widetilde{S}^{2}(t)+2 \eta_{1}(\theta)|\widetilde{S}(t)|\left|\widetilde{N}_{1}(t)\right|\right)= \\
=\sqrt{\left\langle H_{1} x(t), x(t)\right\rangle} \sqrt{h_{00}}\left\langle\left(\begin{array}{cc}
\frac{\alpha_{1}}{D_{1}}\left(S^{0}-\lambda_{1}\right) D \zeta_{1}(\theta) & \eta_{1}(\theta) \\
\eta_{1}(\theta) & 0
\end{array}\right)\left(\begin{array}{c}
|\widetilde{S}(t)| \\
\left|\widetilde{N}_{1}(t)\right|
\end{array}\right),\left(\begin{array}{c}
|\widetilde{S}(t)| \\
\left|\widetilde{N}_{1}(t)\right|
\end{array}\right)\right\rangle .
\end{gathered}
$$

В силу леммы 1 справедливо неравенство

$$
\left\langle\left(\begin{array}{cc}
\frac{\alpha_{1}}{D_{1}}\left(S^{0}-\lambda_{1}\right) D \zeta_{1}(\theta) & \eta_{1}(\theta) \\
\eta_{1}(\theta) & 0
\end{array}\right)\left(\begin{array}{c}
|\widetilde{S}(t)| \\
\left|\widetilde{N}_{1}(t)\right|
\end{array}\right),\left(\begin{array}{c}
|\widetilde{S}(t)| \\
\left|\widetilde{N}_{1}(t)\right|
\end{array}\right)\right\rangle \leqslant \xi_{1}\left\langle H_{1}\left(\begin{array}{c}
\widetilde{S}(t) \\
\widetilde{N}_{1}(t)
\end{array}\right),\left(\begin{array}{c}
\widetilde{S}(t) \\
\widetilde{N}_{1}(t)
\end{array}\right)\right\rangle,
$$

где $\xi_{1}$ определяется по формуле (4.9). Отсюда вытекает оценка

$$
2\left\langle H_{1} x(t), F_{1}(x(t))\right\rangle \leqslant q_{1}\left\langle H_{1} x(t), x(t)\right\rangle^{3 / 2} \leqslant q_{1} V^{3 / 2}(t, x),
$$

где $q_{1}$ определено в (4.8).

Из установленной оценки и неравенства (5.7) при $t \in\left[0, t^{*}\right]$ получим оценку

$$
\begin{aligned}
\frac{d}{d t} V(t, x) \leqslant-\left(c_{1}-2 e^{\kappa_{1} \tau_{1} / 2} \sqrt{h_{11} \nu_{1}} \alpha_{1} \theta\right)\left\langle H_{1} x(t), x(t)\right\rangle+ & \\
& +q_{1} V^{3 / 2}(t, x)-\kappa_{1} \int_{t-\tau_{1}}^{t}\left\langle K_{1}(t-s) x(s), x(s)\right\rangle d s .
\end{aligned}
$$

Отсюда, учитывая обозначение (4.8) и определение (4.2) функционала $V(t, x)$, нетрудно получить неравенство

$$
\frac{d}{d t} V(t, x) \leqslant-\delta_{1} V(t, x)+q_{1} V^{3 / 2}(t, x) .
$$

Из данной оценки, используя неравенство Гронуолла (см., например, [11]), установим оценку

$$
V(t, x) \leqslant \frac{V(0, \psi) e^{-\delta_{1} t}}{\left(1-\frac{q_{1}}{\delta_{1}} \sqrt{V(0, \psi)}\right)^{2}}
$$

Отсюда получим неравенство

$$
\left\langle H_{1} x(t), x(t)\right\rangle \leqslant \frac{V(0, \psi) e^{-\delta_{1} t}}{\left(1-\frac{q_{1}}{\delta_{1}} \sqrt{V(0, \psi)}\right)^{2}} .
$$

Поскольку

$$
\widetilde{S}^{2}(t) \leqslant s_{1}\left\langle H_{1} x(t), x(t)\right\rangle, \quad \widetilde{N}_{1}^{2}(t) \leqslant n_{1}\left\langle H_{1} x(t), x(t)\right\rangle,
$$

где $s_{1}$ и $n_{1}$ определены в (4.10), из последнего неравенства нетрудно установить справедливость оценок (4.13) и (4.14) при $t \in\left[0, t^{*}\right]$. В частности, из оценок (4.12) и (4.13) следуют неравенства

$$
|\widetilde{S}(t)| \leqslant \theta, \quad t \in\left[0, t^{*}\right], \quad\left|\widetilde{S}\left(t^{*}\right)\right|<\theta .
$$

Получили противоречие с тем, что $\left|\widetilde{S}\left(t^{*}\right)\right|=\theta$. Тем самым, оценки (4.13) и (4.14) справедливы при всех $t \in\left[0, \tau_{1}\right]$, при этом будут выполнены неравенства

$$
|\widetilde{S}(t)| \leqslant \theta, \quad t \in\left[0, \tau_{1}\right], \quad\left|\widetilde{S}\left(\tau_{1}\right)\right|<\theta .
$$

Далее рассмотрим промежуток $t \in\left[\tau_{1}, 2 \tau_{1}\right]$. Проводя те же самые рассуждения, что и в случае $t \in\left[0, \tau_{1}\right]$, установим справедливость неравенств (4.13) и (4.14) при $t \in\left[\tau_{1}, 2 \tau_{1}\right]$. Применяя метод математической индукции, получим оценки (4.13) и (4.14) при $t \in\left[k \tau_{1},(k+1) \tau_{1}\right], k \in \mathbb{N}$. В силу произвольности $k \in \mathbb{N}$ отсюда вытекает справедливость неравенств (4.13) и (4.14) при всех $t>0$. Теорема 2 доказана. 
6. Оценки скорости сходимости к нетривиальному положению равновесия в случае произвольного $n$. В данном разделе будем предполагать, что выполнено условие $\lambda_{1}<S^{0}$, функция $p_{1}(S)$ является непрерывно дифференцируемой в некоторой окрестности точки $S=\lambda_{1}$ и

$$
p_{1}^{\prime}\left(\lambda_{1}\right)>0, \quad p_{i}\left(\lambda_{1}\right)<\frac{D_{i}}{\alpha_{i}}, \quad i=2, \ldots, n .
$$

В этом случае в силу теоремы 1 нетривиальное положение равновесия (1.4) системы (1.1) является асимптотически устойчивым.

Поскольку исследование устойчивости положения равновесия (1.4) системы (1.1) эквивалентно исследованию устойчивости нулевого решения системы (2.13), для системы (2.13) рассмотрим следующую начальную задачу:

$$
\left\{\begin{array}{l}
\frac{d}{d t} y(t)=\mathbf{A} y(t)+\sum_{i=1}^{n} \mathbf{B}_{i} y\left(t-\tau_{i}\right)+\mathbf{F}(y(t))+\mathbf{G}\left(y\left(t-\tau_{1}\right), \ldots, y\left(t-\tau_{n}\right)\right), \\
y(t)=\psi(t), \quad t \in\left[-\tau_{\max }, 0\right], \quad y(+0)=\psi(0), \quad \tau_{\max }=\max \left\{\tau_{1}, \ldots, \tau_{n}\right\},
\end{array}\right.
$$

где $y(t), \mathbf{A}, \mathbf{B}_{i}, i=1, \ldots, n, \mathbf{F}(y(t)), \mathbf{G}\left(y\left(t-\tau_{1}\right), \ldots, y\left(t-\tau_{n}\right)\right)$ определены в (2.4), (2.14)-(2.17),

$$
\psi(t)=\left(\begin{array}{c}
\psi_{0}(t) \\
\psi_{1}(t) \\
\psi_{2}(t) \\
\vdots \\
\psi_{n}(t)
\end{array}\right), \quad\left\{\begin{array}{l}
\psi_{0}(t)=\varphi_{0}(t)-\lambda_{1}, \\
\psi_{1}(t)=\varphi_{1}(t)-\frac{\alpha_{1}}{D_{1}}\left(S^{0}-\lambda_{1}\right) D, \\
\psi_{2}(t)=\varphi_{2}(t), \ldots, \psi_{n}(t)=\varphi_{n}(t),
\end{array}\right.
$$

$\varphi_{0}(t), \varphi_{1}(t), \ldots, \varphi_{n}(t)$ - непрерывные неотрицательные функции.

Мы получим оценки решений начальной задачи (6.1), характеризующие скорость убывания на бесконечности, и укажем оценки множества притяжения нулевого решения. В силу замены (2.1) из полученных оценок будут вытекать соответствующие результаты для начальной задачи (1.1)(1.2) для произвольного $n$.

При получении оценок будем использовать модифицированный функционал ЛяпуноваКрасовского, построенный по аналогии с функционалом (4.2):

$$
\mathbf{V}(t, y)=\langle\mathbf{H} y(t), y(t)\rangle+\sum_{i=1}^{n} \int_{t-\tau_{i}}^{t}\left\langle\mathbf{K}_{i}(t-s) y(s), y(s)\right\rangle d s,
$$

где

$$
\begin{aligned}
\mathbf{H} & =\left(\begin{array}{cccc}
H_{1} & 0 & \ldots & 0 \\
0 & h_{2} & \ldots & 0 \\
\vdots & \vdots & \ddots & \vdots \\
0 & 0 & \ldots & h_{n}
\end{array}\right), \quad H_{1}=\left(\begin{array}{cc}
h_{00} & h_{01} \\
h_{01} & h_{11}
\end{array}\right), \\
\mathbf{K}_{1}(s) & =\left(\begin{array}{cccc}
K_{1}(s) & 0 & \ldots & 0 \\
0 & 0 & \ldots & 0 \\
\vdots & \vdots & \ddots & \vdots \\
0 & 0 & \ldots & 0
\end{array}\right), \quad K_{1}(s)=e^{-\kappa_{1} s}\left(B_{1}^{*} B_{1}+M_{1}\right), \quad s \in\left[0, \tau_{1}\right], \\
\mathbf{K}_{2}(s) & =\left(\begin{array}{cccc}
\mathbf{0} & 0 & \ldots & 0 \\
0 & k_{2}(s) & \ldots & 0 \\
\vdots & \vdots & \ddots & \vdots \\
0 & 0 & \ldots & 0
\end{array}\right), \quad k_{2}(s)=e^{-\kappa_{2} s} \beta_{2},
\end{aligned}
$$




$$
\mathbf{K}_{n}(s)=\left(\begin{array}{cccc}
\mathbf{0} & 0 & \ldots & 0 \\
0 & 0 & \ldots & 0 \\
\vdots & \vdots & \ddots & \vdots \\
0 & 0 & \ldots & k_{n}(s)
\end{array}\right), \quad k_{n}(s)=e^{-\kappa_{n} s} \beta_{n}, \quad s \in\left[0, \tau_{n}\right] .
$$

Нетрудно заметить, что функционал можно переписать в следующем виде:

$$
\begin{aligned}
\mathbf{V}(t, y)=\left\langle H_{1} x(t), x(t)\right\rangle+ & \sum_{i=2}^{n} h_{i} \widetilde{N}_{i}^{2}(t)+ \\
& +\int_{t-\tau_{1}}^{t}\left\langle K_{1}(t-s) x(s), x(s)\right\rangle d s+\sum_{i=2}^{n} \int_{t-\tau_{i}}^{t} k_{i}(t-s) \widetilde{N}_{i}^{2}(s) d s .
\end{aligned}
$$

Перейдем к описанию величин, входящих в определение функционала (6.5). Так же, как и в случае $n=1$, величины $h_{00}, h_{01}, h_{11}$ определяются по формулам (3.10), число $\kappa_{1}$ определяется из неравенств (3.12).

Пусть величина $c_{1}>0$ определяется из неравенства (4.3). Предположим, что функция $p_{1}(S)$ является дважды непрерывно дифференцируемой, а функции $p_{i}(S), i=2, \ldots, n$, один раз непрерывно дифференцируемы. При $\theta>0$ рассмотрим функции (4.4)

$$
\zeta_{1}(\theta)=\max _{\substack{|s| \leqslant \theta \\ \lambda_{1}+s \geqslant 0}}\left|p_{1}^{\prime \prime}\left(\lambda_{1}+s\right)\right|, \quad \eta_{1}(\theta)=\max _{\substack{|s| \leqslant \theta \\ \lambda_{1}+s \geqslant 0}}\left|p_{1}^{\prime}\left(\lambda_{1}+s\right)\right|,
$$

а также введем следующие функции:

$$
\eta_{i}(\theta)=\max _{\substack{|s| \leqslant \theta \\ \lambda_{1}+s \geqslant 0}}\left|p_{i}^{\prime}\left(\lambda_{1}+s\right)\right|, \quad i=2, \ldots, n .
$$

Пусть $\theta>0$ таково, что выполнено неравенство (4.5):

$$
2 e^{\kappa_{1} \tau_{1} / 2} \sqrt{h_{11} \nu_{1}} \alpha_{1} \theta<c_{1},
$$

где величина $\nu_{1}$ определяется по формуле (4.6), и имеют место следующие неравенства:

$$
\alpha_{i} p_{i}\left(\lambda_{1}+\theta\right)<D_{i}, \quad i=2, \ldots, n .
$$

Так же, как и выше, определим матрицу $M_{1}=M_{1}^{*}>0$ по формуле (4.7).

Далее определим величины $\kappa_{i}>0, i=2, \ldots, n$, из следующих условий:

$$
c_{i}=2\left(D_{i}-e^{\kappa_{i} \tau_{i} / 2} \alpha_{i} p_{i}\left(\lambda_{1}+\theta\right)\right)>0, \quad i=2, \ldots, n .
$$

Пусть $\varepsilon_{i}>0, i=2, \ldots, n$, - такие числа, что выполняется неравенство

$$
\omega_{1}=2 e^{\kappa_{1} \tau_{1} / 2} \sqrt{h_{11} \nu_{1}} \alpha_{1} \theta+\sum_{i=2}^{n} \varepsilon_{i} h_{00}<c_{1}
$$

а величины $h_{i}>0, i=2, \ldots, n$, определяются из неравенств

Наконец, положим

$$
h_{i}>\frac{p_{i}^{2}\left(\lambda_{1}\right)}{c_{i} \varepsilon_{i}}, \quad i=2, \ldots, n .
$$

$$
\beta_{i}=e^{\kappa_{i} \tau_{i} / 2} h_{i} \alpha_{i} p_{i}\left(\lambda_{1}+\theta\right), \quad i=2, \ldots, n .
$$

Функционал (6.5) полностью определен.

Для формулировки основных результатов введем еще некоторые обозначения. Положим

$$
\delta=\min \left\{\left(c_{1}-\omega_{1}\right),\left(c_{2}-\frac{p_{2}^{2}\left(\lambda_{1}\right)}{h_{2} \varepsilon_{2}}\right), \ldots,\left(c_{n}-\frac{p_{n}^{2}\left(\lambda_{1}\right)}{h_{n} \varepsilon_{n}}\right), \kappa_{1}, \kappa_{2}, \ldots, \kappa_{n}\right\}>0 .
$$

Пусть $\sigma>0$ - произвольное положительное число,

$$
q=\sqrt{h_{00}} \max \{\xi, \sigma\},
$$


где

$$
\begin{gathered}
\xi=\frac{h_{01} \eta_{1}(\theta)+\frac{1}{2} h_{11} \varrho(\theta)+\sqrt{h_{11}\left(h_{00} \eta_{1}^{2}(\theta)+h_{01} \varrho(\theta) \eta_{1}(\theta)+\frac{1}{4} h_{11} \varrho^{2}(\theta)\right)}}{\left(h_{00} h_{11}-h_{01}^{2}\right)}, \\
\varrho(\theta)=\left[\frac{\alpha_{1}}{D_{1}}\left(S^{0}-\lambda_{1}\right) D \zeta_{1}(\theta)+\frac{1}{\sigma} \sum_{i=2}^{n} \frac{\eta_{i}^{2}(\theta)}{h_{i}}\right] .
\end{gathered}
$$

Определим величины $s_{1}$ и $n_{1}$ по формулам (4.10):

$$
s_{1}=\frac{h_{11}}{\left(h_{00} h_{11}-h_{01}^{2}\right)}, \quad n_{1}=\frac{h_{00}}{\left(h_{00} h_{11}-h_{01}^{2}\right)} .
$$

Наконец, перейдем к формулировке основных результатов.

Теорема 4. Пусть $\lambda_{1}<S^{0}$, функиия $p_{1}(S)$ является дважды непрерывно дифферениируемой, функиии $p_{i}(S), i=2, \ldots, n$, один раз непрерывно дифферениируемы, $p_{1}^{\prime}\left(\lambda_{1}\right)>0$ и $p_{i}\left(\lambda_{1}\right)<D_{i} / \alpha_{i}$, $i=2, \ldots, n$. Тогда для решения $y(t)=\left(\widetilde{S}(t), \widetilde{N}_{1}(t), \widetilde{N}_{2}(t), \ldots, \widetilde{N}_{n}(t)\right)^{T}$ начальной задачи $(6.1) c$ начальными данными, удовлетворяющими условиям

$$
\begin{gathered}
\max _{t \in\left[-\tau_{1}, 0\right]}\left|\psi_{0}(t)\right| \leqslant \theta, \quad\left|\psi_{0}(0)\right|<\theta, \\
\psi_{0}(t)+\lambda_{1} \geqslant 0, \quad \psi_{1}(t)+\frac{\alpha_{1}}{D_{1}}\left(S^{0}-\lambda_{1}\right) D \geqslant 0, \quad \psi_{i}(t) \geqslant 0, \quad i=2, \ldots, n, \\
\sqrt{\mathbf{V}(0, \psi)}<\frac{\delta}{q}, \quad \frac{\sqrt{s_{1}} \sqrt{\mathbf{V}(0, \psi)}}{\left(1-\frac{q}{\delta} \sqrt{\mathbf{V}(0, \psi)}\right)} \leqslant \theta
\end{gathered}
$$

справедливы оченки

$$
\begin{array}{ll}
|\widetilde{S}(t)| \leqslant \frac{\sqrt{s_{1}} \sqrt{\mathbf{V}(0, \psi)}}{\left(1-\frac{q}{\delta} \sqrt{\mathbf{V}(0, \psi)}\right)} e^{-\delta t / 2}, & t>0, \\
\left|\widetilde{N}_{1}(t)\right| \leqslant \frac{\sqrt{n_{1}} \sqrt{\mathbf{V}(0, \psi)}}{\left(1-\frac{q}{\delta} \sqrt{\mathbf{V}(0, \psi)}\right)} e^{-\delta t / 2}, & t>0, \\
\left|\widetilde{N}_{i}(t)\right| \leqslant \frac{1}{\sqrt{h_{i}}} \frac{\sqrt{\mathbf{V}(0, \psi)}}{\left(1-\frac{q}{\delta} \sqrt{\mathbf{V}(0, \psi)}\right)} e^{-\delta t / 2}, & i=2, \ldots, n, \quad t>0 .
\end{array}
$$

Доказательство теоремы 4 будет приведено в следующем разделе.

Сформулируем результат для системы (1.1), непосредственно вытекающий из теоремы 4. Введем обозначения

$$
\varphi(t)=\left(\begin{array}{c}
\varphi_{0}(t) \\
\varphi_{1}(t) \\
\varphi_{2}(t) \\
\vdots \\
\varphi_{n}(t)
\end{array}\right), \quad y^{*}=\left(\begin{array}{c}
\lambda_{1} \\
\frac{\alpha_{1}}{D_{1}}\left(S^{0}-\lambda_{1}\right) D \\
0 \\
\vdots \\
0
\end{array}\right)
$$

Справедлива следующая теорема.

Теорема 5. Пусть $\lambda_{1}<S^{0}$, функиия $p_{1}(S)$ является дважды непрерывно дифферениируемой, функиии $p_{i}(S), i=2, \ldots, n$, один раз непреръьвно дифференцируемы, $p_{1}^{\prime}\left(\lambda_{1}\right)>0$ и $p_{i}\left(\lambda_{1}\right)<D_{i} / \alpha_{i}$, $i=2, \ldots, n$. Тогда для решения $\left(S(t), N_{1}(t), N_{2}(t), \ldots, N_{n}(t)\right)^{T}$ начальной задачи $(1.1)-(1.2)$ с начальными данными, удовлетворялощими условиям

$$
\max _{t \in\left[-\tau_{1}, 0\right]}\left|\varphi_{0}(t)-\lambda_{1}\right| \leqslant \theta, \quad\left|\varphi_{0}(0)-\lambda_{1}\right|<\theta, \quad \varphi_{i}(t) \geqslant 0, \quad i=0,1,2, \ldots, n
$$




$$
\sqrt{\mathbf{V}\left(0, \varphi-y^{*}\right)}<\frac{\delta}{q}, \quad \frac{\sqrt{s_{1}} \sqrt{\mathbf{V}\left(0, \varphi-y^{*}\right)}}{\left(1-\frac{q}{\delta} \sqrt{\mathbf{V}\left(0, \varphi-y^{*}\right)}\right)} \leqslant \theta
$$

справедливы оченки

$$
\begin{array}{ll}
\left|S(t)-\lambda_{1}\right| \leqslant \frac{\sqrt{s_{1}} \sqrt{\mathbf{V}\left(0, \varphi-y^{*}\right)}}{\left(1-\frac{q}{\delta} \sqrt{\mathbf{V}\left(0, \varphi-y^{*}\right)} e^{-\delta t / 2},\right.} \quad t>0, \\
\left|N_{1}(t)-\frac{\alpha_{1}}{D_{1}}\left(S^{0}-\lambda_{1}\right) D\right| \leqslant \frac{\sqrt{n_{1}} \sqrt{\mathbf{V}\left(0, \varphi-y^{*}\right)}}{\left(1-\frac{q}{\delta} \sqrt{\mathbf{V}\left(0, \varphi-y^{*}\right)}\right)} e^{-\delta t / 2}, & t>0, \\
\left|N_{i}(t)\right| \leqslant \frac{1}{\sqrt{h_{i}}} \frac{\sqrt{\mathbf{V}\left(0, \varphi-y^{*}\right)}}{\left(1-\frac{q}{\delta} \sqrt{\mathbf{V}\left(0, \varphi-y^{*}\right)}\right)} e^{-\delta t / 2}, & i=2, \ldots, n, \quad t>0 .
\end{array}
$$

7. Доказательство теоремы 4. Рассмотрим модифицированный функционал ЛяпуноваКрасовского (6.5). Дифференцируя его вдоль решения начальной задачи (6.1), получим

$$
\begin{aligned}
& \frac{d}{d t} \mathbf{V}(t, y)=\left\langle H_{1} \frac{d}{d t} x(t), x(t)\right\rangle+\left\langle H_{1} x(t), \frac{d}{d t} x(t)\right\rangle+\sum_{i=2}^{n} 2 h_{i} \widetilde{N}_{i}(t) \frac{d}{d t} \widetilde{N}_{i}(t)+ \\
& +\left\langle K_{1}(0) x(t), x(t)\right\rangle-\left\langle K_{1}\left(\tau_{1}\right) x\left(t-\tau_{1}\right), x\left(t-\tau_{1}\right)\right\rangle+\int_{t-\tau_{1}}^{t}\left\langle\frac{d}{d t} K_{1}(t-s) x(s), x(s)\right\rangle d s+ \\
& +\sum_{i=2}^{n} k_{i}(0) \widetilde{N}_{i}^{2}(t)-\sum_{i=2}^{n} k_{i}\left(\tau_{i}\right) \widetilde{N}_{i}^{2}\left(t-\tau_{i}\right)+\sum_{i=2}^{n} \int_{t-\tau_{i}}^{t} \frac{d}{d t} k_{i}(t-s) \widetilde{N}_{i}^{2}(s) d s .
\end{aligned}
$$

Учитывая, что система дифференциальных уравнений в начальной задаче (6.1) может быть записана в виде (2.12), а матрица $K_{1}(s)$ и функции $k_{i}(s), i=2, \ldots, n$, имеют вид (6.2)-(6.4), производную от функционала $\mathbf{V}(t, y)$ можно переписать в виде

$$
\begin{aligned}
& \frac{d}{d t} \mathbf{V}(t, y)=\left\langle\left(\begin{array}{cc}
H_{1} A_{1}+A_{1}^{*} H_{1}+K_{1}(0) & H_{1} B_{1} \\
B_{1}^{*} H_{1} & -K_{1}\left(\tau_{1}\right)
\end{array}\right)\left(\begin{array}{c}
x(t) \\
x\left(t-\tau_{1}\right)
\end{array}\right),\left(\begin{array}{c}
x(t) \\
x\left(t-\tau_{1}\right)
\end{array}\right)\right\rangle+ \\
& +2\left\langle H_{1} x(t), G_{1}\left(x\left(t-\tau_{1}\right)\right)\right\rangle+2\left\langle H_{1} x(t), L(y(t))\right\rangle+2\left\langle H_{1} x(t),\left(F_{1}(x(t))+U(y(t))\right)\right\rangle+ \\
& \quad+\sum_{i=2}^{n} 2 h_{i} \widetilde{N}_{i}(t)\left(-D_{i} \widetilde{N}_{i}(t)+\alpha_{i} p_{i}\left(\lambda_{1}+\widetilde{S}\left(t-\tau_{i}\right)\right) \widetilde{N}_{i}\left(t-\tau_{i}\right)\right)+\sum_{i=2}^{n} \beta_{i} \widetilde{N}_{i}^{2}(t)- \\
& -\sum_{i=2}^{n} e^{-\kappa_{i} \tau_{i}} \beta_{i} \widetilde{N}_{i}^{2}\left(t-\tau_{i}\right)-\kappa_{1} \int_{t-\tau_{1}}^{t}\left\langle K_{1}(t-s) x(s), x(s)\right\rangle d s-\sum_{i=2}^{n} \kappa_{i} \int_{t-\tau_{i}}^{t} k_{i}(t-s) \widetilde{N}_{i}^{2}(s) d s .
\end{aligned}
$$

Введем обозначения

$$
\begin{gathered}
J_{1}(t)=\left\langle\left(\begin{array}{cc}
H_{1} A_{1}+A_{1}^{*} H_{1}+K_{1}(0) & H_{1} B_{1} \\
B_{1}^{*} H_{1} & -K_{1}\left(\tau_{1}\right)
\end{array}\right)\left(\begin{array}{c}
x(t) \\
x\left(t-\tau_{1}\right)
\end{array}\right),\left(\begin{array}{c}
x(t) \\
x\left(t-\tau_{1}\right)
\end{array}\right)\right\rangle+2\left\langle H_{1} x(t), G_{1}\left(x\left(t-\tau_{1}\right)\right)\right\rangle, \\
J_{2}(t)=2\left\langle H_{1} x(t), L(y(t))\right\rangle, \\
J_{3}(t)=\sum_{i=2}^{n} 2 h_{i} \widetilde{N}_{i}(t)\left(-D_{i} \widetilde{N}_{i}(t)+\alpha_{i} p_{i}\left(\lambda_{1}+\widetilde{S}\left(t-\tau_{i}\right)\right) \widetilde{N}_{i}\left(t-\tau_{i}\right)\right)+\sum_{i=2}^{n} \beta_{i} \widetilde{N}_{i}^{2}(t)-\sum_{i=2}^{n} e^{-\kappa_{i} \tau_{i}} \beta_{i} \widetilde{N}_{i}^{2}\left(t-\tau_{i}\right), \\
J_{4}(t)=2\left\langle H_{1} x(t),\left(F_{1}(x(t))+U(y(t))\right)\right\rangle .
\end{gathered}
$$

Тогда тождество (7.1) принимает вид 


$$
\begin{aligned}
\frac{d}{d t} \mathbf{V}(t, y)=J_{1}(t)+J_{2}(t)+J_{3}(t) & +J_{4}(t)- \\
& \quad-\kappa_{1} \int_{t-\tau_{1}}^{t}\left\langle K_{1}(t-s) x(s), x(s)\right\rangle d s-\sum_{i=2}^{n} \kappa_{i} \int_{t-\tau_{i}}^{t} k_{i}(t-s) \tilde{N}_{i}^{2}(s) d s .
\end{aligned}
$$

Вначале предположим, что $t \in\left[0, \tau_{\min }\right]$, где $\tau_{\min }=\min \left\{\tau_{1}, \ldots, \tau_{n}\right\}$.

1. Оценим $J_{1}(t)$. Поскольку $t \in\left[0, \tau_{\min }\right]$, то $t-\tau_{1} \leqslant 0$. Следовательно, в силу условия (6.14) имеем

$$
\left|\widetilde{S}\left(t-\tau_{1}\right)\right|=\left|\psi_{0}\left(t-\tau_{1}\right)\right| \leqslant \theta .
$$

Точно так же, как и в случае $n=1$, установим справедливость неравенства

$$
J_{1}(t) \leqslant-\left(c_{1}-2 e^{\kappa_{1} \tau_{1} / 2} \sqrt{h_{11} \nu_{1}} \alpha_{1} \theta\right)\left\langle H_{1} x(t), x(t)\right\rangle,
$$

где $c_{1}$ определено в (4.3), $\nu_{1}$ определено в (4.6).

2. Оценим $J_{2}(t)$. Учитывая явный вид (2.10) вектор-функции $L(y(t))$, получим

$$
J_{2}(t)=2\left\langle\left(\begin{array}{cc}
h_{00} & h_{01} \\
0 & 0
\end{array}\right)\left(\begin{array}{c}
\widetilde{S}_{(t)} \\
\widetilde{N}_{1}(t)
\end{array}\right),\left(\begin{array}{c}
l(y(t)) \\
0
\end{array}\right)\right\rangle=2\left\langle\widetilde{H}_{0} x(t), L(y(t))\right\rangle,
$$

где

$$
\widetilde{H}_{0}=\left(\begin{array}{cc}
h_{00} & h_{01} \\
0 & 0
\end{array}\right) .
$$

Следовательно, в силу формулы (2.10) имеем

$$
\begin{gathered}
J_{2}(t)=-2 \sum_{i=2}^{n}\left\langle\widetilde{H}_{0} x(t),\left(\begin{array}{c}
p_{i}\left(\lambda_{1}\right) \widetilde{N}_{i}(t) \\
0
\end{array}\right)\right\rangle \leqslant 2 \sum_{i=2}^{n}\left\|\widetilde{H}_{0} x(t)\right\|\left\|\left(\begin{array}{c}
p_{i}\left(\lambda_{1}\right) \widetilde{N}_{i}(t) \\
0
\end{array}\right)\right\|= \\
=2 \sum_{i=2}^{n}\left\|\widetilde{H}_{0} x(t)\right\| p_{i}\left(\lambda_{1}\right) \widetilde{N}_{i}(t) \leqslant \sum_{i=2}^{n}\left(\varepsilon_{i}\left\|\widetilde{H}_{0} x(t)\right\|^{2}+\frac{p_{i}^{2}\left(\lambda_{1}\right)}{\varepsilon_{i}} \widetilde{N}_{i}^{2}(t)\right)= \\
=\sum_{i=2}^{n} \varepsilon_{i}\left\langle\widetilde{H}_{0}^{*} \widetilde{H}_{0} x(t), x(t)\right\rangle+\sum_{i=2}^{n} \frac{p_{i}^{2}\left(\lambda_{1}\right)}{\varepsilon_{i}} \widetilde{N}_{i}^{2}(t) .
\end{gathered}
$$

Нетрудно видеть, что имеет место неравенство

$$
\left\langle\widetilde{H}_{0}^{*} \widetilde{H}_{0} x, x\right\rangle \leqslant h_{00}\left\langle H_{1} x, x\right\rangle, \quad x \in \mathbb{R}^{2} .
$$

Отсюда получим оценку

$$
J_{2}(t) \leqslant \sum_{i=2}^{n} \varepsilon_{i} h_{00}\left\langle H_{1} x(t), x(t)\right\rangle+\sum_{i=2}^{n} \frac{p_{i}^{2}\left(\lambda_{1}\right)}{\varepsilon_{i}} \widetilde{N}_{i}^{2}(t) .
$$

3. Оценим $J_{3}(t)$ :

$$
J_{3}(t) \leqslant-\sum_{i=2}^{n}\left(2 h_{i} D_{i}-\beta_{i}-\frac{\left(h_{i} \alpha_{i} p_{i}\left(\lambda_{1}+\widetilde{S}\left(t-\tau_{i}\right)\right)\right)^{2}}{e^{-\kappa_{i} \tau_{i}} \beta_{i}}\right) \widetilde{N}_{i}^{2}(t) .
$$

Поскольку $t \in\left[0, \tau_{\min }\right]$, то $t-\tau_{i} \leqslant 0, i=2, \ldots, n$. Следовательно, в силу условия (6.14) имеем

$$
\left|\widetilde{S}\left(t-\tau_{i}\right)\right|=\left|\psi_{0}\left(t-\tau_{i}\right)\right| \leqslant \theta .
$$

Учитывая монотонное возрастание функций $p_{i}(S), i=2, \ldots, n$, и обозначения (6.9), отсюда нетрудно установить неравенство

$$
J_{3}(t) \leqslant-\sum_{i=2}^{n} 2\left(D_{i}-e^{\kappa_{i} \tau_{i} / 2} \alpha_{i} p_{i}\left(\lambda_{1}+\theta\right)\right) h_{i} \widetilde{N}_{i}^{2}(t) .
$$


В силу обозначений (6.7) это неравенство запишется в виде

$$
J_{3}(t) \leqslant-\sum_{i=2}^{n} c_{i} h_{i} \widetilde{N}_{i}^{2}(t)
$$

4. Оценим $J_{4}(t)$. Учитывая явный вид $(2.6)$ и (2.11) вектор-функций $F_{1}(x(t))$ и $U(y(t))$, получим оценку

$$
\begin{array}{r}
J_{4}(t)=2\left\langle H_{1}^{1 / 2} x(t), H_{1}^{1 / 2}\left(F_{1}(x(t))+U(y(t))\right)\right\rangle \leqslant 2\left\|H_{1}^{1 / 2} x(t)\right\|\left\|H_{1}^{1 / 2}\left(F_{1}(x(t))+U(y(t))\right)\right\|= \\
=2 \sqrt{\left\langle H_{1} x(t), x(t)\right\rangle} \sqrt{h_{00}}\left(\left|f_{a}(x(t))+f_{b}(x(t))+u(y(t))\right|\right),
\end{array}
$$

где

$$
\begin{aligned}
f_{a}(x(t)) & =\frac{\alpha_{1}}{D_{1}}\left(S^{0}-\lambda_{1}\right) D\left(p_{1}\left(\lambda_{1}+\widetilde{S}(t)\right)-p_{1}\left(\lambda_{1}\right)-p_{1}^{\prime}\left(\lambda_{1}\right) \widetilde{S}(t)\right) \\
f_{b}(x(t)) & =\left(p_{1}\left(\lambda_{1}+\widetilde{S}(t)\right)-p_{1}\left(\lambda_{1}\right)\right) \widetilde{N}_{1}(t) \\
u(y(t)) & =-\sum_{i=2}^{n}\left(p_{i}\left(\lambda_{1}+\widetilde{S}(t)\right)-p_{i}\left(\lambda_{1}\right)\right) \widetilde{N}_{i}(t) .
\end{aligned}
$$

В силу условия (6.14) имеем $|\widetilde{S}(0)|=\left|\psi_{0}(0)\right|<\theta$. Покажем, что неравенство $|\widetilde{S}(t)|<\theta$ также будет выполнено при $t \in\left[0, \tau_{\min }\right]$. Предположим, напротив, что существует такая точка $t^{*} \in\left(0, \tau_{\min }\right]$, что $|\widetilde{S}(t)|<\theta$ при $t \in\left[0, t^{*}\right)$ и $\left|\widetilde{S}\left(t^{*}\right)\right|=\theta$. Тогда при $t \in\left[0, t^{*}\right]$ по аналогии со случаем, когда $n=1$, установим справедливость неравенств

$$
\begin{gathered}
\left|p_{1}\left(\lambda_{1}+\widetilde{S}(t)\right)-p_{1}\left(\lambda_{1}\right)-p_{1}^{\prime}\left(\lambda_{1}\right) \widetilde{S}(t)\right| \leqslant \frac{1}{2} \zeta_{1}(\theta) \widetilde{S}^{2}(t), \\
\left|p_{1}\left(\lambda_{1}+\widetilde{S}(t)\right)-p_{1}\left(\lambda_{1}\right)\right| \leqslant \eta_{1}(\theta)|\widetilde{S}(t)|, \quad\left|p_{i}\left(\lambda_{1}+\widetilde{S}(t)\right)-p_{i}\left(\lambda_{1}\right)\right| \leqslant \eta_{i}(\theta)|\widetilde{S}(t)|, \quad i=2, \ldots, n,
\end{gathered}
$$

где $\zeta_{1}(\theta)$ и $\eta_{1}(\theta)$ определены в $(4.4), \eta_{i}(\theta), i=2, \ldots, n$, определены в (6.6). Отсюда следует оценка

$$
\begin{gathered}
J_{4}(t) \leqslant \sqrt{\left\langle H_{1} x(t), x(t)\right\rangle} \sqrt{h_{00}}\left(\frac{\alpha_{1}}{D_{1}}\left(S^{0}-\lambda_{1}\right) D \zeta_{1}(\theta) \widetilde{S}^{2}(t)+\right. \\
\left.+2 \eta_{1}(\theta)|\widetilde{S}(t)|\left|\widetilde{N}_{1}(t)\right|+\sum_{i=2}^{n} 2 \eta_{i}(\theta)|\widetilde{S}(t)|\left|\widetilde{N}_{i}(t)\right|\right) \leqslant \\
\leqslant \sqrt{\left\langle H_{1} x(t), x(t)\right\rangle} \sqrt{h_{00}}\left(\left[\frac{\alpha_{1}}{D_{1}}\left(S^{0}-\lambda_{1}\right) D \zeta_{1}(\theta)+\frac{1}{\sigma} \sum_{i=2}^{n} \frac{\eta_{i}^{2}(\theta)}{h_{i}}\right] \widetilde{S}^{2}(t)+\right. \\
\left.+2 \eta_{1}(\theta)|\widetilde{S}(t)|\left|\widetilde{N}_{1}(t)\right|+\sigma \sum_{i=2}^{n} h_{i} \widetilde{N}_{i}^{2}(t)\right) .
\end{gathered}
$$

В силу леммы 1 справедливо неравенство

$$
\left[\frac{\alpha_{1}}{D_{1}}\left(S^{0}-\lambda_{1}\right) D \zeta_{1}(\theta)+\frac{1}{\sigma} \sum_{i=2}^{n} \frac{\eta_{i}^{2}(\theta)}{h_{i}}\right] \widetilde{S}^{2}(t)+2 \eta_{1}(\theta)|\widetilde{S}(t)|\left|\widetilde{N}_{1}(t)\right| \leqslant \xi\left\langle H_{1} x(t), x(t)\right\rangle,
$$

где $\xi$ определяется по формулам (6.12)-(6.13). Отсюда вытекает оценка

$$
J_{4}(t) \leqslant \sqrt{\left\langle H_{1} x(t), x(t)\right\rangle} \sqrt{h_{00}}\left(\xi\left\langle H_{1} x(t), x(t)\right\rangle+\sigma \sum_{i=2}^{n} h_{i} \widetilde{N}_{i}^{2}(t)\right) .
$$


Из данной оценки, учитывая обозначение (6.11) и определение (6.5) функционала $\mathbf{V}(t, y)$, получим неравенство

$$
J_{4}(t) \leqslant q \sqrt{\left\langle H_{1} x(t), x(t)\right\rangle}\left(\left\langle H_{1} x(t), x(t)\right\rangle+\sum_{i=2}^{n} h_{i} \widetilde{N}_{i}^{2}(t)\right) \leqslant q \mathbf{V}^{3 / 2}(t, y) .
$$

Итак, используя представление (7.2) производной функционала $\mathbf{V}(t, y)$ и полученные оценки (7.3)-(7.6), при $t \in\left[0, t^{*}\right]$ приходим к следующему неравенству:

$$
\begin{aligned}
\frac{d}{d t} \mathbf{V}(t, y) \leqslant-\left(c_{1}-2 e^{\kappa_{1} \tau_{1} / 2} \sqrt{h_{11} \nu_{1}} \alpha_{1} \theta-\sum_{i=2}^{n} \varepsilon_{i} h_{00}\right)\left\langle H_{1} x(t), x(t)\right\rangle- & \\
-\sum_{i=2}^{n}\left(c_{i}-\frac{p_{i}^{2}\left(\lambda_{1}\right)}{h_{i} \varepsilon_{i}}\right) h_{i} \widetilde{N}_{i}^{2}(t)+q \mathbf{V}^{3 / 2}(t, y)- & -\kappa_{1} \int_{t-\tau_{1}}^{t}\left\langle K_{1}(t-s) x(s), x(s)\right\rangle d s-\sum_{i=2}^{n} \kappa_{i} \int_{t-\tau_{i}}^{t} k_{i}(t-s) \widetilde{N}_{i}^{2}(s) d s .
\end{aligned}
$$

Отсюда, учитывая обозначения (6.8) и (6.10), нетрудно получить неравенство

$$
\frac{d}{d t} \mathbf{V}(t, y) \leqslant-\delta \mathbf{V}(t, y)+q \mathbf{V}^{3 / 2}(t, y)
$$

Из данной оценки, используя неравенство Гронуолла (см., например, [11]), выводим оценку

$$
\mathbf{V}(t, y) \leqslant \frac{\mathbf{V}(0, \psi) e^{-\delta t}}{\left(1-\frac{q}{\delta} \sqrt{\mathbf{V}(0, \psi)}\right)^{2}}
$$

Отсюда нетрудно получить оценки (6.16)-(6.18) при $t \in\left[0, t^{*}\right]$. В частности, из оценок (6.15) и (6.16) следуют неравенства

$$
|\widetilde{S}(t)| \leqslant \theta, \quad t \in\left[0, t^{*}\right], \quad\left|\widetilde{S}\left(t^{*}\right)\right|<\theta .
$$

Получили противоречие с тем, что $\left|\widetilde{S}\left(t^{*}\right)\right|=\theta$. Тем самым, оценки (6.16)-(6.18) будут справедливы при всех $t \in\left[0, \tau_{\min }\right] ;$ при этом будут выполнены неравенства

$$
|\widetilde{S}(t)| \leqslant \theta, \quad t \in\left[0, \tau_{\min }\right], \quad\left|\widetilde{S}\left(\tau_{\min }\right)\right|<\theta .
$$

Далее рассмотрим промежуток $t \in\left[\tau_{\min }, 2 \tau_{\min }\right]$. Проводя те же самые рассуждения, что и в случае $t \in\left[0, \tau_{\min }\right]$, установим справедливость неравенств (6.16)-(6.18) при $t \in\left[\tau_{\min }, 2 \tau_{\min }\right]$. Применяя метод математической индукции, получим оценки (6.16)-(6.18) при $t \in\left[k \tau_{\min },(k+1) \tau_{\min }\right], k \in \mathbb{N}$. В силу произвольности $k \in \mathbb{N}$ отсюда будет следовать справедливость неравенств (6.16)-(6.18) при всех $t>0$.

Теорема 4 доказана.

Автор выражает благодарность профессору Г. В. Демиденко за внимание к работе.

\section{СПИСОК ЛИТЕРАТУРЫ}

1. Демиденко Г. В. Матричные уравнения. - Новосибирск: Изд-во Новосиб. ун-та, 2009.

2. Демиденко Г. В., Матвеева И. И. Асимптотические свойства решений дифференциальных уравнений с запаздывающим аргументом// Вестн. НГУ. Сер. мат. мех. информ. -2005 . -5 , № 3. - С. 20-28.

3. Демиденко Г. В., Матвеева И. И. Устойчивость решений дифференциальных уравнений с запаздывающим аргументом и периодическими коэффициентами в линейных членах// Сиб. мат. ж. -2007. - 48, № 5. - C. 1025-1040.

4. Красовский Н. Н. Некоторые задачи теории устойчивости движения. - М.: ГИФМЛ, 1959.

5. Матвеева И. И. Оценки решений одного класса систем нелинейных дифференциальных уравнений с запаздывающим аргументом// Сиб. ж. индустр. мат. - 2013. - 16, № 3. - С. 122-132.

6. Скворцова M. А. Устойчивость решений в модели хищник-жертва с запаздыванием// Мат. заметки СВФУ. - 2016. - 23, № 2. - С. 108-120. 
7. Скворцова M. A. Асимптотическая устойчивость положений равновесия и оценки решений в одной модели заболевания// Динам. сист. - 2017. - 7 (35), № 3. - С. 257-274.

8. Скворцова M. А. Оценки решений в модели хищник-жертва с запаздыванием// Изв. Иркутск. гос. ун-та. Сер. мат. - 2018. - 25. - С. 109-125.

9. Сквориова M. A. Об оценках решений в модели хищник-жертва с двумя запаздываниями// Сиб. электрон. мат. изв. - 2018. - 15. - С. 1697-1718.

10. Скворцова M. А. Асимптотические свойства решений в модели взаимодействия популяций с несколькими запаздываниями// Мат. заметки СВФУ. - 2019. - 26, № 4.

11. Хартман Ф. Обыкновенные дифференциальные уравнения. - М.: Мир, 1970.

12. Хусаинов Д. Я., Иванов А. Ф., Кожаметов А. Т. Оценки сходимости решений линейных стационарных систем дифференциально-разностных уравнений с постоянным запаздыванием// Диффер. уравн. - 2005. - 41, № 8. - C. 1137-1140.

13. Mondie S., Kharitonov V. L. Exponential estimates for retarded time-delay systems: LMI approach// IEEE Trans. Automat. Control. - 2005. - 50, № 2. - P. 268-273.

14. Wolkowicz G. S. K., Xia H. Global asymptotic behavior of a chemostat model with discrete delays// SIAM J. Appl. Math. - 1997. — 57, № 4. - P. 1019-1043.

Скворцова Мария Александровна

Институт математики им. С. Л. Соболева СО РАН, Новосибирск;

Новосибирский государственный университет

E-mail: sm-18-nsu@yandex.ru 